\title{
Morphology and Electrophysiological Properties of Immunocytochemically Identified Rat Dopamine Neurons Recorded in vitro
}

\author{
Anthony A. Grace and Shao-Pii Onn \\ Departments of Behavioral Neuroscience and Psychiatry, Center for Neuroscience, University of Pittsburgh, Pittsburgh, \\ Pennsylvania 15260
}

In vitro intracellular recordings were made from neurons in the rat midbrain slice. Two neuronal types could be distinguished in dopamine-containing (DA) midbrain regions based on electrophysiological criteria. One neuron type exhibited short duration action potentials ( $<1.5 \mathrm{msec}$ ), could fire at high frequencies $(>10 \mathrm{~Hz}$ ), and exhibited either phasic or burst firing patterns. This neuron did not exhibit tyrosine hydroxylase immunoreactivity. A second neuronal type exhibited a unique set of electrophysiological properties, which included (1) a spontaneous pacemaker-like depolarizing potential, (2) a highly regular firing pattern, (3) long duration ( $>2$ msec) action potentials, and (4) a high (i.e., depolarized) spike threshold. This neuron was consistently double labeled using intracellular staining and immunocytochemical localization of the catecholamine-specific enzyme tyrosine hydroxylase, and thus represented the DA neuronal type. Midbrain DA neurons stained with Lucifer yellow could be separated into 3 classes based on their location and morphology: (1) fusiform neurons with laterally projecting dendrites in the dorsal substantia nigra zona compacta region, (2) multipolar cells with laterally and ventrally projecting dendrites in the ventral substantia nigra zona compacta, and (3) neurons with fusiform and multipolar somata and radially projecting dendrites in the ventral tegmental area. The dendrites also exhibited spine-like protrusions and ended with specialized forked processes.

Spontaneously firing DA cells recorded in vitro had a number of distinguishing electrophysiological characteristics in common with those of DA neurons recorded in vivo, such as the presence of a slow depolarizing potential driving spike activity and a characteristic depolarized spike threshold (approximately $-36 \mathrm{mV}$ ). However, in contrast to that found in vivo, the DA cells characterized here exhibited substantially higher input resistances and fired spontaneously in a very regular pacemaker pattern. Burst firing was not observed. Spike activity was apparently dependent on 4 depolarizing events: (1) a voltage-dependent TTX-sensitive slow depolarization, (2) a cobalt-sensitive low threshold depolarization that was activated during the rebound from brief membrane

\footnotetext{
Received Jan. 13, 1989; revised Mar. 8, 1989; accepted Apr. 6, 1989.

We wish to thank Mr. Jeffrey Hollerman and Ms. Michele Pucak for suggestions in the preparation of this manuscript. This work was supported by USPHS MH42217, MH30915, NS19608, and the Scottish Rite Schizophrenia Research Program, N.M.J., U.S.A. S.P.O. is supported by a Tourette's Foundation fellowship. A.A.G. is a Sloan fellow.

Correspondence should be addressed to Anthony A. Grace at the above address. Copyright (c) 1989 Society for Neuroscience $0270-6474 / 89 / 103463-19 \$ 02.00 / 0$
}

hyperpolarizations, (3) high threshold dendritic calcium spikes which gave rise to the spike afterhyperpolarization, and (4) a high threshold initial segment sodium spike. These depolarizations were modulated by several processes, including a 4-aminopyridine-insensitive delayed repolarization, an instantaneous and time-dependent anomalous rectifler, and an afterhyperpolarlzation. Although low threshold depolarizations and rebound action potentials could be triggered by the membrane repolarization following small membrane hyperpolarizations, comparatively larger hyperpolarizations attenuated this rebound activation, thereby suppressing anodal break excitation.

These experiments provide a basis for the identification of DA neurons in the in vitro brain slice preparation by their characteristic electrophysiological properties. By examining the morphology and regulation of activity in this neurochemically defined cell class, information pertaining to the functioning of DA neurons will be garnered, which may eventually lead to alternate avenues of therapeutic intervention in DArelated psychiatric disorders.

Dysfunctions of the midbrain dopaminergic (DA) systems have been implicated in the etiology of several clinical disorders, such as Parkinson's disease (Hornykiewicz, 1963, 1966) and schizophrenia (Snyder, 1973; Matthysse, 1973). These findings have led to extensive research into the function of this small group of brain stem neurons using several experimental preparations. Among the approaches used to investigate this system, in vivo electrophysiological recordings from identified DA-containing neurons have played a major role in determining the mode of action of a variety of psychotogenic and therapeutic drugs, such as amphetamine (Groves et al., 1975; Bunney and Aghajanian, 1976) and the neuroleptics (Bunney and Grace, 1978; Chiodo and Bunney, 1983; White and Wang, 1983; Grace and Bunney, 1986). However, in order to derive valid neuropharmacological information about the mechanisms of drug action through the recording of neuronal activity, it is first necessary to establish that the cells recorded contain the neurotransmitter under study. By combining electrophysiological characterizations of putative midbrain DA neurons with histochemical confirmation of their neurochemical identity (Bunney et al., 1973; Grace and Bunney, $1980,1983 a$ ), a set of physiological criteria was established for identifying DA neurons recorded in situ in the anesthetized rat. In this way, the in vivo studies of DA neuron activity have provided information about the range of responses and discharge patterns that DA neurons are capable of expressing in the intact organism and how this activity could be modulated 
by drugs administered to the whole organism. However, in vivo recordings are somewhat limited in their ability to identify the membrane processes underlying neuronal activity or in distinguishing between pharmacological responses mediated directly and those acting through afferent systems. Thus, we initiated a series of studies to investigate the membrane properties that underlie the activity of midbrain DA neurons using the in vitro brain slice preparation, which is more amenable to this level of analysis.

One problem that arises in using the in vitro preparation for investigating DA neuron electrophysiology is again confirming that the recordings were made from identified DA neurons. Unfortunately, many of the identification criteria advanced in the in vivo experiments could not be applied to the in vitro preparation, such as the ability to antidromically activate neurons from their termination sites (Deniau et al., 1978; Guyenet and Aghajanian, 1978; Thierry et al., 1979; Preston et al., 1981; Grace and Bunney, 1980, 1983a). Other electrophysiological criteria used to identify DA neurons in vivo, such as firing pattern, appear to be sufficiently altered in vitro (Brodie and Dunwiddie, 1987; Grace, 1987; Shepard and Bunney, 1988) to render this classification criterion inadequate for this preparation. Identification based on anatomical location is complicated by many factors, including (1) the observation that DA neurons in the zona compacta lie within a thin sheet of cells and are intermixed with non-DA neurons (Guyenet and Crane, 1981) and (2) a study reporting a significant probability of impaling and staining non-DA neurons in the substantia nigra zona compacta during in vitro recordings (Bargas et al., 1988). Therefore, the first step in this investigation was to identify, in the in vitro slice preparation, which physiologically defined neuronal type in the substantia nigra region contained DA as a neurotransmitter.

The next stage of analysis involved the development of a model of DA neuron action potential generation in terms of the cell's spike generating sites and their role in promoting neuronal discharge. This would be important for understanding such cellular properties as (1) the manner by which excitation is spread throughout the neuron, (2) the sites at which synaptic input may be positioned to exert a significant influence on cell activity, (3) how modulation of active membrane sites within a neuron may influence its firing rate or pattern, and (4) whether zones of current influx are related to release of neurotransmitters from dendritic sites. This type of information may be highly relevant for discerning the functional implications of DA cell electrophysiological activity, given that these cells are known to contain and release DA from their dendritic as well as their axon terminal regions (Geffen et al., 1976; Korf et al., 1976), and there is evidence suggesting that dendritic DA release is likely to be modulated by factors different from those regulating the release of DA from nerve terminals (Chéramy et al., 1981, 1985; Romo et al., 1986). Thus, details of DA neuron spike generation may be relevant for understanding how DA cell activity states and firing patterns are regulated and, furthermore, can provide insights into the relationship between these spike-generating zones and the compartmentalization of function within this neuronal type. Part of these data has been presented at symposia (Grace, 1987, 1988; Grace and Onn, 1988a, b).

\section{Materials and Methods}

Sprague-Dawley albino male rats obtained from Zivic-Miller Laboratories were used in all experiments and were handled in accordance with the procedures outlined in the Guide for the Care and Use of
Laboratory Animals published by the USPHS. The specific protocols used were approved by the University of Pittsburgh Animal Care Committee. Rats weighing between 225 and $350 \mathrm{gm}$ were deeply anesthetized with sodium pentobarbital (Nembutal; $50 \mathrm{mg} / \mathrm{kg}$ i.p.) before decapitation. After removal of the surrounding cranium, the brain was rapidly excised, and a $4 \mathrm{~mm}$ thick section was cut perpendicular to the brain axis using an Activational Systems Rat Brain Matrix (RBM-4000C) to guide the cut. A block from the midbrain region (2200-4700 $\mu$ m anterior to the interaural line; Paxinos and Watson, 1982) was then sectioned in ice-cold Ringer's solution (124 mM NaCl, $5 \mathrm{~mm} \mathrm{KCl,} 1.2 \mathrm{mM} \mathrm{KH}_{2} \mathrm{PO}_{4}$, $2.4 \mathrm{mM} \mathrm{CaCl}_{2}, 1.3 \mathrm{mM} \mathrm{MgSO}_{4}, 26 \mathrm{mM} \mathrm{NaHCO}_{3}, 10 \mathrm{~mm}$ glucose, and saturated with $95 \%: 5 \% \mathrm{O}_{2}: \mathrm{CO}_{2}$ ) to $400 \mu \mathrm{m}$ thickness using a Vibratome (Oxford). The brain sections were placed in continuously oxygenated Ringer's solution at room temperature for $1-2 \mathrm{hr}$ before recording. The slices were then transferred to a submersion-type chamber maintained at $37^{\circ} \mathrm{C}$ and superfused with oxygenated Ringer's adjusted to a flow rate of $2-3 \mathrm{ml} / \mathrm{min}$, as previously described (Llinás and Sugimori, 1980a, b; Grace and Llinás, 1985).

Signals captured by the electrodes were amplified by an adjacent head stage amplifier connected to a preamplifier (NeuroData IR-283). Current was injected across a bridge circuit, with electrode voltage and current injection amplitudes monitored on an oscilloscope (Hitachi V-1050F). These output signals were also digitized at $44 \mathrm{kHz}$ per channel using a NeuroData 4-channel Neurocorder (Neurodata DR-484) and stored on VHS videotapes (VHS T-120) for subsequent off-line analysis. Spike potentials were analyzed using a CED1401 Intelligent Laboratory Interface (Cambridge Electronic Design, Cambridge, UK) outfitted with 8 megabytes of Massram. This unit was interfaced with a Compaq 386/ 20 microcomputer equipped with a $130 \mathrm{MB}$ high-speed fixed disk drive and an Amdek 1280 high-resolution monochrome monitor for display, measurement, and analysis of the digitized data. Hard copy displays of long spike trains were produced by directing the output of data captured by the CED1401 Massram to a Gould RS3400 3-channel chart recorder. In order to assure accurate representation of spike peaks and fast transients, the digitized data stored in the CED1401 Massram were converted into analog form by running the CED1401 internal digital-toanalog converters at $1 / 100$ of the rate used for data capture, thus assuring that the output transients would remain within the limited dynamic range of the chart recorder pens. Details of shorter duration spike trains or responses to individual current pulses were obtained by directing data through the Compaq computer for output onto a Hewlett-Packard 7475A plotter using drafting pens and polyester film to generate accurate plots of the stored data.

Electrodes were pulled from $1 \mathrm{~mm}$ diameter Omegadot (WPI, New Haven, CT) borosilicate glass tubing using a Flaming/Brown P-80/PC electrode puller. The glass pipettes were then filled with one of the following solutions: (1) $3.0 \mathrm{~m}$ potassium acetate for intracellular recording (impedance, 45-75 M $\Omega$ ) or (2) 10\% Lucifer yellow (Sigma; Stewart, 1978) in distilled water (impedance, $80-150 \mathrm{M} \Omega$ ) for combined recording and staining. Current was injected across a bridge circuit built into the preamplifier. Only neurons evidencing stable penetrations were used for electrophysiological analyses and dye injection, with stability defined as resting potentials greater than $-50 \mathrm{mV}$, action potential amplitudes greater than $65 \mathrm{mV}$, and temporally uniform input resistances typically greater than $80 \mathrm{M} \Omega$. Putative DA neurons were tentatively identified during intracellular recording by their location, the presence of a slow pacemaker-like depolarization (slow depolarization) preceding spontaneously occurring action potentials, and the long duration ( $>2 \mathrm{msec}$ ) action potentials (Grace and Bunney, 1983a). Intracellular staining was performed using electrodes filled with Lucifer yellow dissolved in distilled water, and tyrosine hydroxylase immunoreactivity was localized using an indirect immunofluorescence technique. Cells were stained intracellularly using electrodes filled with $10 \%$ Lucifer yellow (Sigma), with the dye ejected by applying l-2 nA continuous hyperpolarizing current to the electrode. This constant current was interrupted by 50 -msec $2 \mathrm{nA}$ depolarizing current pulses delivered at $5-6 \mathrm{~Hz}$ to prevent clogging of the electrode tip (Grace and Llinás, 1985). Cells were injected with the dye for 5-20 min to obtain details of distal processes for use in morphological studies and for $1-4$ min in the double-labeling studies to circumvent bleedthrough of Lucifer yellow fluorescence at longer wavelengths. Slices used in double-labeling studies were then placed in fixative $(4 \%$ paraformaldehyde in $0.1 \mathrm{M}$ phosphate buffer, $\mathrm{pH}, 7.4$, containing $10 \%$ sucrose) overnight at $4^{\circ} \mathrm{C}$. Serial sections $20 \mu \mathrm{m}$ in thickness were cut on a cryostat (Reichert) and collected onto albumin-coated slides. The Lucifer yellow-injected cells 
were localized in the sections using a Leitz Orthoplan II epifluorescence microscope equipped with a Leitz D filter cube (excitation: band pass $355-425 \mathrm{~nm}$; dichromatic mirror: RKP $455 \mathrm{~nm}$; suppression: low pass $460 \mathrm{~nm}$ ). Typically, the entire soma of the stained neuron was located in a single section, with distal processes contained in adjacent sections. Slices used for morphological investigations were fixed overnight in $10 \%$ phosphate-buffered formalin ( $\mathrm{pH} 7.4$ ), washed in buffered saline, and cleared by placing them in $100 \%$ dimethylsulfoxide (DMSO) for $20 \mathrm{~min}$ (Grace and Llinás, 1985). Slices were then transferred to microscope slides and mounted in DMSO for viewing with the fluorescence microscope.

Tyrosine hydroxylase-like immunoreactivity was localized using immunocytochemical detection (Sternberger et al., 1970) on sections of brain slices containing the Lucifer yellow-labeled cells and their processes. The slides were incubated with tyrosine hydroxylase antisera (Eugene Tech; $1000 \times$ dilution with $0.1 \mathrm{M}$ phosphate buffer containing $5 \%$ normal goat serum and $0.3 \%$ Triton $\mathrm{X}-100$ ) for $2 \mathrm{~d}$ in a moisturized chamber maintained at $4^{\circ} \mathrm{C}$, followed by overnight incubation with rhodamine-conjugated goat antirabbit IgG (Sigma; $60 \times$ dilution with same buffer), also at $4^{\circ} \mathrm{C}$. Sections were washed repeatedly, then airdried and mounted in glycerol. Double-labeled cells were first photographed using a Leitz N2 filter (excitation: band pass $530-560 \mathrm{~nm}$; dichromatic mirror: RKP580 nm; suppression: low pass $580 \mathrm{~nm}$ ), which passes fluorescence from the rhodamine label and attenuates that from the Lucifer yellow. The cells were photographed again after changing to the Leitz D filter, which will preferentially pass Lucifer yellow fluorescence and block fluorescence arising from the rhodamine label.

The pharmacology of the membrane potential changes observed was determined by the addition of specific ion channel blockers [ $1-2 \mu \mathrm{M}$ TTX to block sodium channels; $2-4 \mathrm{~mm}$ tetraethylammonium (TEA) to block some potassium channels; $1-3 \mathrm{~mm}$ cobalt to block calcium influx; and 4-aminopyridine (4-AP): $5 \mathrm{~mm}$ and $20 \mathrm{~mm}$ to block preferentially the transient type potassium channels]. These compounds were added to the Ringer's at a constant rate set by a microprocessorcontrolled peristaltic pump (Haake-Büchler model MCP2500), which added the solutions directly to the mixing compartment of the slice chamber. This permitted rapid and precise control of the composition of the medium. For studies involving the administration of cobalt, Tris buffer was substituted for the bicarbonate buffer to avoid precipitation of the cation, and the solution was oxygenated using $99.5 \% \mathrm{O}_{2}: 0.5 \%$ $\mathrm{CO}_{2}$.

All values given in text are mean $\pm \mathrm{SD}$.

\section{Results}

\section{Cell identification}

Intracellular recordings were made from neurons in the zona compacta region of the substantia nigra and in the ventral tegmental area. Neurons recorded within these midbrain regions could be readily classified into two broad subtypes based on their morphological and electrophysiological characteristics. Only one of these neuron types was labeled as containing tyrosine hydroxylase-like immunoreactivity.

Non-DA neurons. One neuronal type recorded within these regions rarely exhibited spontaneous spike activity and, on depolarization, fired large amplitude, fast action potentials (60$75 \mathrm{mV}$, duration: $1.3 \pm 0.3 \mathrm{msec}$, mean $\pm \mathrm{SD}$ ). The action potential threshold was typical of mammalian neurons (i.e., -53 $\pm 4 \mathrm{mV}$ ) and did not vary appreciably with changes in membrane potential. The input resistance of the neurons averaged $135 \pm 50 \mathrm{M} \Omega(n=12)$. Depolarization of the membrane from resting potentials elicited repetitively firing spikes (Fig. $1 A$ ). However, depolarization of the membrane from more hyperpolarized potentials (i.e., negative to $-75 \mathrm{mV}$ ) elicited a low threshold, TTX-resistant depolarization with an accompanying burst of action potentials. The interval between consecutive spikes occurring within a burst was very brief (i.e., 3-5 msec; Fig. $1 B$ ). Intracellular injection of Lucifer yellow into neurons displaying these physiological characteristics consistently labeled large ( $30 \mu \mathrm{m}$ diameter or greater) multipolar neurons lo- cated in the substantia nigra zona compacta, the nearby reticular formation, and the zona reticulata. These cells had numerous dendritic processes arising directly from the soma, including dendrites extending into regions dorsal to the substantia nigra. In 3 cases, neurons of this class that had been stained by intracellular injection of Lucifer yellow were examined for the presence of tyrosine hydroxylase-like immunoreactivity using a rhodaminc-labcled antibody. Nonc of these stained ncurons wcrc found to exhibit double labeling for tyrosine hydroxylase immunoreactivity. Furthermore, the morphological, histochemical, and electrophysiological characteristics found for this neuron type were not consistent with those of histochemically identified DA neurons described in anatomical studies (Schwyn and Fox, 1974; Hökfelt et al., 1976; Lindvall and Björklund, 1974, 1978; Juraska et al., 1977) or in vivo intracellular recording and staining of physiologically identified DA cells (Grace and Bunney, 1980, 1983a, b; Grace, 1987, 1988; Tepper et al., 1987).

$D A$ neuron identification. A physiologically distinct neuronal type was also recorded in the zona compacta region of the substantia nigra and in the ventral tegmental area. These neurons exhibited action potentials similar to those reported for identified DA neurons recorded in vivo; i.e., action potentials (1) were long in duration (>2 msec), (2) had comparatively depolarized thresholds for activation (i.e., -30 to $-45 \mathrm{mV}$ ), (3) wcre triggered by a voltage-dependent slow depolarization, and (4) were followed by a prominent afterhyperpolarization (Fig. 1C). In every case where neurons displaying these electrophysiological properties were stained by intracellular injection of the dye Lucifer yellow, the neurons also demonstrated double labeling for tyrosine hydroxylase-like immunoreactivity $(n=7)$, both in the substantia nigra (Fig. $2 A$ ) and in the ventral tegmental area (Fig. 2B). Of course, tyrosine hydroxylase is not specific for DA neurons, since this enzyme is also located in other catecholamine-synthesizing neurons, i.e., those containing norepinephrine or epinephrine. However, studies have failed to localize the specific noradrenergic enzyme dopamine- $\beta$-hydroxylase (Fuxe et al., 1971; Swanson and Hartman, 1975) or the adrenergic enzyme phenylethanolamine $\mathrm{N}$-methyltransferase (Kitahama et al., 1988) to neuronal somata in this brain region. Furthermore, rhodamine-labeled cells were only observed in midbrain regions known to contain DA cells (i.e., the zona compacta of the substantia nigra and the ventral tegmental area). Thus, these experiments confirm that this class of physiologically defined neurons was of the DA-containing type.

One problem often encountered with double-labeling studies is distinguishing the fluorescence of the injected dye from the fluorescence of the immunocytochemical stain. In these experiments, care was taken to ensure that comparatively small amounts of Lucifer yellow were injected into the neuron, thereby limiting the observable emission spectrum of this dye. Thus, in double-labeling studies, Lucifer yellow was injected for periods of only 1-4 min to facilitate its discrimination from the immunocytochemical fluorescent label. Under these circumstances, only neurons displaying the characteristic DA cell physiological pattern demonstrated double labeling for Lucifer yellow and tyrosine hydroxylase immunoreactivity. Bleedthrough of the Lucifer yellow dye across the rhodamine filter was not a confounding variable in these experiments because (1) non-DA neurons in the zona reticulata or reticular formation injected with an equivalent amount of Lucifer yellow did not exhibit significant levels of fluorescence when examined with the rhodamine filter, and (2) the regions of double-labeled neurons that 

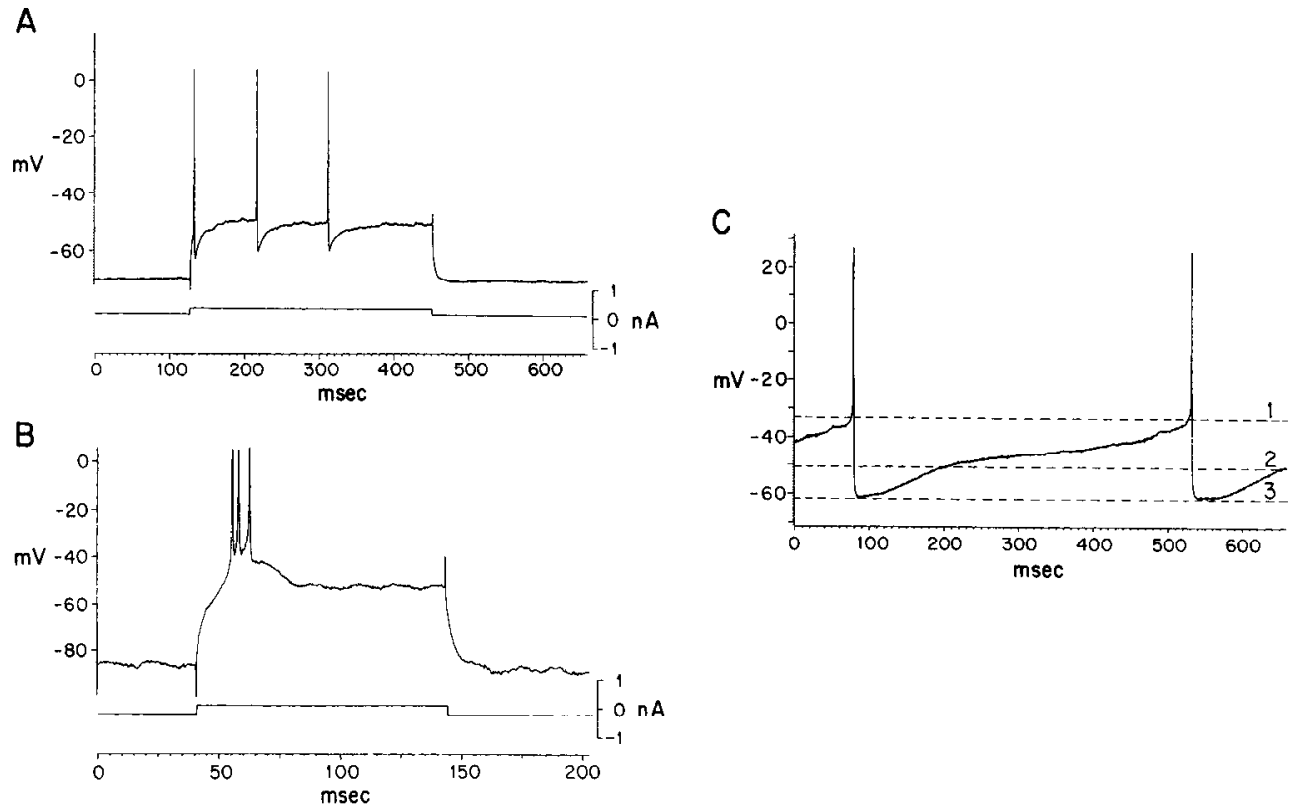

Figure 1. Based on their electrophysiological characteristics, at least two neuronal types located in the DA-containing regions of the rat midbrain slice could be distinguished. One neuron type could be identified by its short duration spikes and its firing pattern. At resting membrane potentials, this cell type exhibited repetitive firing of action potentials in response to a depolarizing pulse $(A)$. On the other hand, hyperpolarization of the membrane of this neuron caused the depolarizing pulse to trigger a low threshold depolarization and a rebound burst of action potentials (B). Neurons with these electrophysiological characteristics did not show the presence of tyrosine hydroxylase-like immunoreactivity in double-labeling studies and hence were not representative of the DA-containing neurons in this region. $C$, The membrane potential changes underlying spontaneous action potential generation in the other class of midbrain neurons recorded in vitro were much like those observed for identified DA neurons in vivo. Spike activity was driven by a slow depolarization, depolarizing the membrane from its resting state (dashed line 2) to that of its characteristically high spike threshold (dashed line 1), in this case, approximately $-33 \mathrm{mV}$. The action potentials were followed by a prominent afterhyperpolarization (dashed line 3), which hyperpolarized the neuron below resting potential and decayed into the onset of the next slow depolarization. Neurons with this electrophysiological pattern were consistently labeled for tyrosine hydroxylase-like immunoreactivity (Fig. 2).

showed the highest level of Lucifer yellow fluorescence usually were distinct from those parts of the neuron that showed the most intense levels of rhodamine fluorescence (Fig. 2). If the fluorescence observed under the rhodamine filter was due to bleedthrough from the Lucifer yellow stain, then the brightest areas of the cell under each filter setting would be identical.

\section{DA neuron morphology}

The morphological characteristics of DA neurons were studied by injecting the highly fluorescent dye Lucifer yellow into neurons displaying the characteristic electrophysiological properties outlined for DA neuron identification. Morphological analyses werc carricd out on 21 DA ncurons injected with Lucifer yellow. The labeled neurons showed a distinct morphology, consisting of medium-sized somata that gave rise to between 2 and 6 thick major dendrites. These sparsely branching dendrites typically exhibited their first bifurcation at distances of $50 \mu \mathrm{m}$ or more from the soma and extended for distances of up to $1200 \mu \mathrm{m}$. From this sample of injected cells, 3 morphological types of DA neurons could be distinguished (Fig. 3): (1) multipolar neurons (20-35 $\mu$ m diameter) in the ventral zona compacta, which had 2-4 major dendrites extending in medial and lateral directions, in addition to 1-3 dendrites extending ventrally into the zona reticulata, (2) fusiform neurons (15-25 $\mu \mathrm{m}$ diameter) in the dorsal portion of the zona compacta, which had 2-5 major dendrites emanating from the soma and extending in medial and lateral directions but remaining within the confines of the zona compacta, and (3) fusiform and multipolar neurons in the ventral tegmental area, which had 3-5 major dendrites projecting radially from the soma. The thin $(0.5 \mu \mathrm{m}$ diameter $)$ axon typically arose from a major dendrite or somatic appendage (Fig. $4 A$ ) and, for substantia nigra neurons, traveled in a medioventral direction within the zona compacta and along the border between the zona compacta and zone reticulata before turning in an anterior direction at the midline. Dendrites typically exhibited spine-like protrusions that occurred at irregular intervals, ranged in length from 5 to $50 \mu \mathrm{m}$, and were $0.5-2 \mu \mathrm{m}$ in diameter (Fig. $4 B$ ). These spinules were particularly prominent on the dendrites of ventral tegmental DA neurons. In each case where the dendrites of DA neurons could be followed to their termination, they were observed to end in a tuft of $2-4$ fine $(0.2-0.8 \mu \mathrm{m}$ diameter) recursive processes that were seen to encompass a circumscribed region of tissue (Fig. $4 \mathrm{C}$ ). In general, this morphology is consistent with morphological studies of identified dopaminergic neurons in these brain regions (Rinvik and Grofova, 1970; Gulley and Wood, 1971; Schwyn and Fox, 1974; Lindvall and Björklund, 1974, 1978; Hökfelt et al., 1976; Juraska et al., 1977; Groves and Linder, 1983).

\section{$D A$ neuron spike generation}

Most DA neurons recorded intracellularly in vitro were spontaneously active, with firing rates ranging between 1 and $7 \mathrm{~Hz}$ (average $=3 \pm 2.5 \mathrm{~Hz} ; n=52$ ). Spontaneous action potential discharge was preceded and apparently driven by a slow depolarization (amplitude $=22 \pm 3 \mathrm{mV}$, duration $=295 \pm 84$ msec, depending on firing frequency; $n=35$ ). The slow depo- 

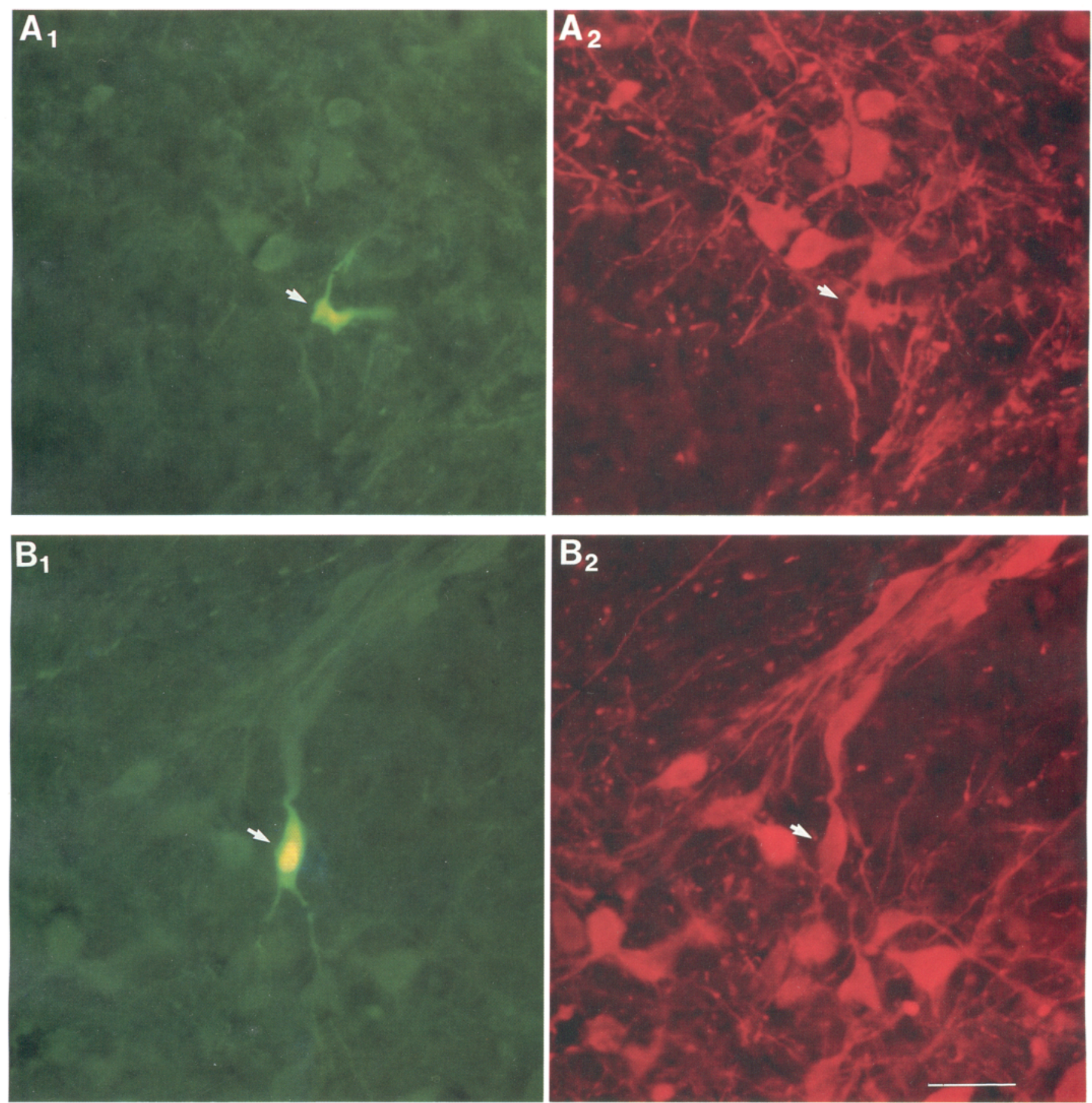

Figure 2. One class of midbrain neurons could be distinguished electrophysiologically based on the presence of a slow depolarization and a pacemaker-like firing pattern. These cells consistently demonstrated the presence of tyrosine hydroxylase-like immunoreactivity, as shown by labeling cells with these unique physiological characteristics by intracellular injection of the dye Lucifer yellow, followed by immunocytochemical localization of tyrosine hydroxylase-like immunoreactivity using antibodies tagged with a rhodamine (red fluorescent) label. $A$, A multipolar neuron in the substantia nigra injected with Lucifer yellow (arrow, $A_{l}$ ) also demonstrated the presence of tyrosine hydroxylase-like immunoreactivity (arrow, $\left.A_{2}\right) B$, A bipolar neuron in the ventral tegmental region that exhibited electrophysiological characteristics similar to those described above was also stained with Lucifer yellow (arrow, $B_{l}$ ) and demonstrated tyrosine hydroxylase-like immunoreactivity $\left(\right.$ arrow, $\left.B_{2}\right)$. Scale bar, $50 \mu \mathrm{m}$.

larization may play a significant role in maintaining spontaneous action potential generation in DA neurons, since it depolarizes the membrane potential from its resting levels $(-57 \pm 4 \mathrm{mV}$; $n=35$; range: -52 to $-67 \mathrm{mV}$ ) to the depolarized spike threshold of DA neurons (Fig. $1 C$ ). The resting potential of spontaneously firing DA neurons was difficult to establish precisely because of the absence of a stable membrane potential in these pacemaker-like neurons but was estimated as the inflection in the membrane potential occurring between the offset of the afterhyperpolarization and the initiation of the next slow depolarization. Spontaneously occurring action potentials were triggered at membrane potentials averaging $-36 \pm 4 \mathrm{mV}$ as 


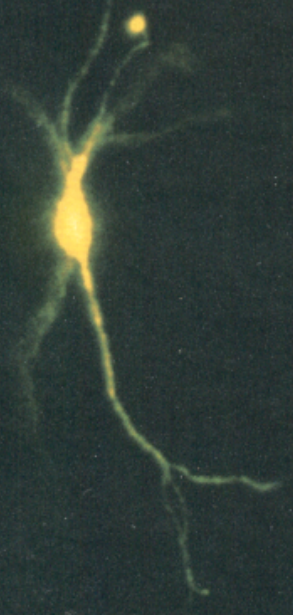

$-$ 
measured at the soma $(n=74)$, which is similar to that of DA neurons recorded in vivo (Grace and Bunney, 1983a). Action potentials were characteristically long in duration, with an average spike duration of $2.7 \pm 0.5 \mathrm{msec}$ (range: $1.8-3.5 \mathrm{msec} ; n$ $=81$ ). The amplitudes of the DA neuron action potentials varied with membrane potential, with an average spike height of 77 $\pm 4 \mathrm{mV}$ (range $=65-92 \mathrm{mV}, n=70$ ) measured from the spike peak to the beginning of the afterhyperpolarization. This type of measurement was necessary to circumvent the problems of measuring spike height using the pre-spike membrane potential as baseline because of the presence of the slow depolarization preceding the spike. DA neuron action potentials were followed by a prominent afterhyperpolarization, which hyperpolarized the cell before the onset of the next slow depolarization.

Although many of the characteristic electrophysiological properties of DA neurons recorded in vitro were similar to those observed during in vivo recordings from identified DA neurons, 2 significant differences in their physiology were noted: (1) DA neurons recorded in vitro had much higher input resistances, and (2) unlike the irregular single spiking or burst firing patterns reported for spontaneously firing DA neurons recorded in vivo (Grace and Bunney, 1984a, b), DA cells recorded in vitro only fired spikes in a very regular, pacemaker-like firing pattern.

\section{Input resistance}

The input resistance of the DA cells recorded was determined by plotting the amplitudes of the hyperpolarizing current pulses injected into the cell against the resultant membrane voltage deflections produced. The input resistance measured in DA neurons was found to be nonlinear over the range of current injection amplitudes tested. The peak input resistance measured in the linear region of the resistance plot (i.e., near the resting potential) was $168 \pm 61 \mathrm{M} \Omega$ (Fig. $5 \mathrm{~A}$ : range: $80-320 \mathrm{M} \Omega, n=$ 38 ), which was about 5 times higher than that reported for DA neurons recorded in vivo (i.e., $31 \pm 7.4 \mathrm{M} \Omega$, range $=18-45 \mathrm{M} \Omega$ ) (Grace and Bunney, 1980, 1983a). During intracellular injection of long (e.g., > $200 \mathrm{msec}$ ) hypcrpolarizing current pulses, a slowly developing sag in the membrane voltage was observed. This is illustrated as a decrease in the slope of the current/voltage plot at long time points (i.e., a decrease in the steady-state input resistance) below that corresponding to the peak input resistance measured. Plotting both the peak and steady-state input resistances showed that these measures deviated from linearity as well as from each other (Fig. 5A). This apparent decrease in input resistance during hyperpolarization of the membrane shows that this event is similar to the anomalous rectifier described previously (Katz, 1949; Adrian, 1969).

\section{Firing pattern}

A second characteristic difference in the physiology of DA neurons recorded in vitro versus those described for DA cells in vivo pertains to their firing pattern. DA ncurons recorded in vivo fired in either an irregular single spiking mode or, on depolarization, in a burst firing pattern (Bunney et al., 1973; Grace and Bunney, 1984a, b). In contrast, DA cells recorded in vitro fired exclusively in a highly regular pacemaker-like pattern. Depolarization of DA neurons in vitro resulted in an increased rateof-rise of the slow depolarization and, hence, an increased firing rate but did not alter the very regular pacemaker firing pattern (Fig. $5 B$ ). The state of the electrode balance was monitored during polarization of the membrane to ensure that the membrane potential observed on the oscilloscope accurately reflected the membrane potential of the DA cell soma. Burst firing was not observed in any of the identified DA neurons recorded in vitro. Furthermore, a firing pattern resembling the burst firing pattern described for DA neurons recorded in vivo (Grace and Bunney, 1984b) or for putative DA neurons recorded in vitro (Ncdcrgaard ct al., 1988a, b) could not be induced in identified DA neurons in this preparation by any of the current injection and membrane polarization parameters examined in this study.

\section{$T T X$-sensitive membrane potentials}

Slow depolarization. In spontaneously firing DA neurons, the slow depolarization is observed as a slowly developing depolarization of the membrane from resting potentials. The slow depolarization averaged $22 \mathrm{mV} \pm 3 \mathrm{mV}(n=35)$, which was sufficient to depolarize the DA neuron membrane from resting potentials to the comparatively high spike threshold found for these neurons in vitro (i.e., $-36 \mathrm{mV}$ ) as well as in vivo $(-41$ $\mathrm{mV}$; Grace and Bunney, 1984a). The slow depolarization can be triggered alone by small depolarizations of the DA neuron. Larger depolarizations caused the slow depolarization to further depolarize the cell sufficiently to trigger an action potential (Fig. $6 A, B)$. The slow depolarization can also be triggered as a component of the rebound depolarization occurring at the termination of brief membrane hyperpolarizations. Thus, injection of small hyperpolarizing current pulses into quiescent DA neurons will cause a rebound depolarization, which can then trigger spike discharge (Fig. $6 D_{1}$ ). The slow depolarization also can be inactivated by briefly hyperpolarizing the membrane. Thus, injection of short-duration hyperpolarizing current pulscs during a spontaneous slow depolarization resets the membrane potential to baseline levels (Grace and Bunney, 1984a), as will the AHP following an action potential (Figs. 1C, 6B). The duration

Figure 3. The morphology of DA neurons was analyzed by injecting physiologically identified midbrain DA neurons with the highly fluorescent dye Lucifer yellow. The labeled cells had medium-sized somata that gave rise to 2-6 sparsely branching dendrites. These cells could be divided into 3 subclasses based on their location and morphology. $A$, DA neurons in the ventral regions of the substantia nigra zona compacta had multipolar somata. This cell had 4 major dendrites extending laterally from the soma, which remained within the zona compacta. In addition, 1 dendrite extended ventrally into regions deep within the zona reticulata. $B$, DA neurons in the more dorsal regions of the substantia nigra had fusiform somata. This neuron had 5 major dendrites extending mediolaterally from the soma, all of which remained entirely within the confines of the zona compacta. $C$, The cell body of this ventral tegmental DA neuron was multipolar in shape, with 5 major dendrites extending from the soma. The dendrites typically projected in a radial array from the soma in this region, apparently depending on the orientation of the DA cell body within the tegmentum. Midline is to the right in $(A)$ and $(B)$ and to the left in $(C)$. Scale bars, $75 \mu \mathrm{m}$ in $A$ and $C$, and $50 \mu \mathrm{m}$ in $B$. 

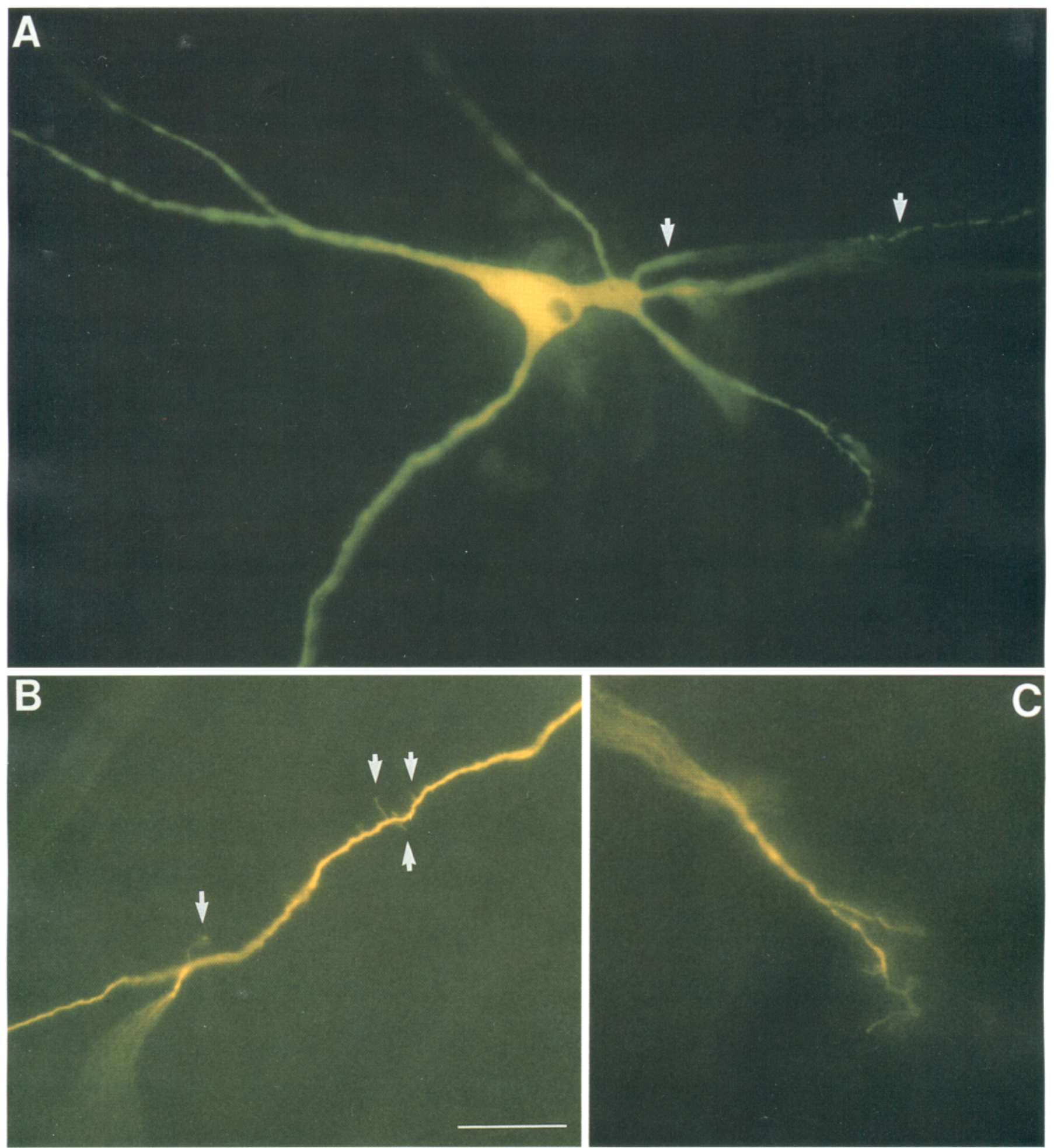

Figure 4. DA neurons labeled by intracellular injection of Lucifer yellow exhibited several unique morphological characteristics. A, In each case where this structure could be identified, the thin, $0.5 \mu \mathrm{m}$ diameter axon (arrows) was observed to arise from a major dendrite or a somatic appendage rather than directly from the soma. $B$, DA neuron dendrites exhibited infrequent spine-like protrusions that extended 3-15 $\mu \mathrm{m}$ from the dendritic shaft (arrows). These protrusions were particularly prominent in labeled DA neurons in the ventral tegmental area. $C$, In each case where the dendrite could be followed to its termination site within the plane of the slice, the dendrite ended in a fork-like tuft. This consisted of recurrent, thin processes that appeared to surround a section of the underlying midbrain, possibly forming a basket around non-DA cells in this region. Many of the fine recurrent processes in this figure are not within the plane of focus of the primary dendrite. Scale bar, $20 \mu \mathrm{m}$. 
of the slow depolarization often will outlast the depolarizing stimulus that triggered it. Thus, short-duration depolarizing pulses will still elicit a slow depolarization and an action potential in DA neurons even if the pulse is terminated before the spike threshold is attained (Fig. 6C). These findings provide additional evidence that this pacemaker-like depolarization exhibits voltage-dependent properties (Grace and Bunney, 1984a). The slow depolarization is strongly influenced by the temperature of the slice preparation, with temperatures below $37^{\circ} \mathrm{C}$ decreasing the rate of activation of the slow depolarization. Indeed, very few spontaneously firing DA neurons could be recorded when the temperature of the preparation fell below $35^{\circ} \mathrm{C}$.

The slow depolarization component of the rebound depolarization triggered in DA cells cannot be attenuated by administering calcium blockers. Thus, the addition of the calcium blocker cobalt ( $2 \mathrm{mM}$ ) to the Ringer's (containing $1.0 \mathrm{mM}$ calcium in Tris buffer in place of the bicarbonate buffer) blocked activation of the rebound spike following a hyperpolarizing current pulse but failed to attenuate the entire rebound response (Fig. $6 D_{1,2}$ ). After cobalt administration, the amplitude of the control rebound depolarization could not be restored regardless of the amount of conditioning hyperpolarization used. Subsequent administration of the sodium blocker TTX $\left(1 \times 10^{-6} \mathrm{M}\right.$ final concentration) to the Ringer's blocked activation of the remaining cobalt-insensitive rebound depolarization (Fig. $6 D_{3}$ ). Thus, blocking calcium channels with cobalt revealed an isolated rebound slow depolarization that could be blocked by TTX. Furthermore, blockade of this depolarization with TTX also eliminated the spontaneous or depolarization-induced membrane potential oscillations underlying the pacemaker firing pattern.

High threshold sodium spikes. Blockade of calcium channels by the admistration of 1-2 mM cobalt chloride into the Ringer's caused a gradual decrease in the amplitude of the action potential and afterhyperpolarization, which culminated in the blockade of spontaneous and depolarization-induced action potential firing (Fig. 7, $A, B$ ). Thus, after cobalt treatment, depolarizations similar to those eliciting action potentials in untreated neurons were ineffective in eliciting active spiking. However, much larger depolarizations resulted in the triggering of large amplitude (42 $\pm 15 \mathrm{mV} ; n=19$; range: $20-60 \mathrm{mV}$ ) cobalt-insensitive spikes (Fig. $7 C$ ). These spikes were characterized by their fast time course $(0.9 \pm 0.2 \mathrm{msec}$ duration; $n=19$; range: $0.5-1.3 \mathrm{msec})$ and their high threshold $(-33 \pm 15 \mathrm{mV} ; n=23$; range: -15 to $-60 \mathrm{mV}$ ). Both the threshold and amplitude of these fast spikes were highly dependent on the membrane potential, as reflected in the comparatively large SDs reported for measures of mean spike amplitude and threshold. Furthermore, although DA neuron action potentials generally exhibit a minimum interspike interval of $>40 \mathrm{msec}$, larger depolarizations of cobalttreated DA neurons elicited these fast spikes at comparatively high frequencies (e.g., 10-15 msec interspike intervals, Fig. 7C). Without cobalt administration, depolarizing DA neurons from resting potentials could not trigger isolated cobalt-resistant spikes-instead, only full amplitude (i.e., >70 mV) action potentials were elicited. However, similar fast spikes could be triggered in DA neurons in normal Ringer's following large hyperpolarizations. In these cases, the fast spikes were triggered at much more hyperpolarized spike thresholds (Fig. $7 E$ ). Both the slow depolarization and the fast spikes were blocked by administering TTX (Fig. 7F). In contrast to the full amplitude
A

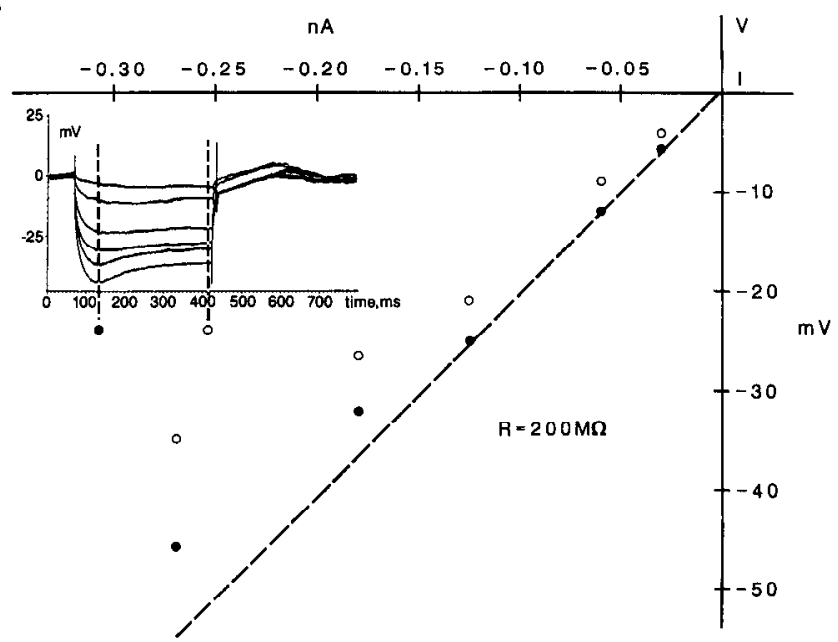

B

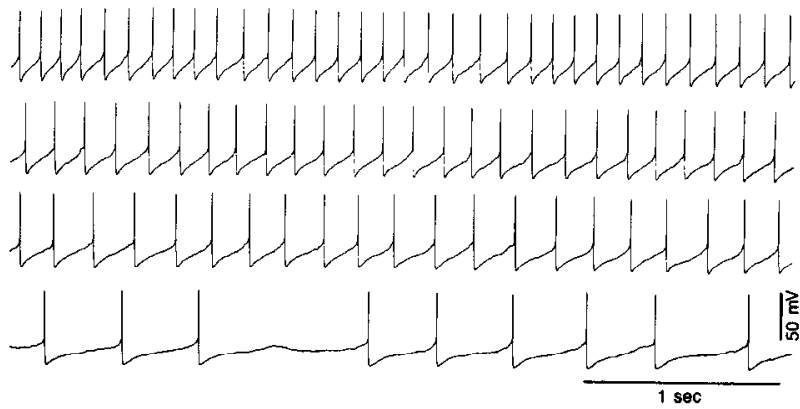

Figure 5. $A$, Input resistance of DA neurons recorded in vitro. The input resistance of this nonfiring DA neuron was calculated by plotting the peak membrane voltage deflections (solid circles) and the steadystate voltage deflections from resting potential (open circles) obtained during the injection of constant current hyperpolarizing pulses (see inset). DA neurons had significantly higher input resistances in the in vitro preparation than observed in vivo. In this case, the input resistance was $200 \mathrm{M} \Omega$. Both the instantaneous and time-dependent components of the anomalous rectification were observed, as shown by the deviation of the peak input resistance regression line from linearity and the deflection of the steady state from the peak input resistances, respectively. $B$, Firing pattern of DA neurons recorded in vitro. Unlike DA neurons recorded in vivo, identified DA cells in the in vitro preparation fired exclusively in a highly regular, pacemaker pattern, and demonstrated little variation in interspike interval over time. Furthermore, depolarization (top 2 traces, $0.05 \mathrm{nA}$ and $0.1 \mathrm{nA}$ ) or hyperpolarization (bottom) trace, $-0.07 \mathrm{nA}$ ) of the DA neurons from baseline (third trace from top) only changed the frequency of spike discharge without altering the firing pattern of the DA neuron.

DA neuron action potential, the fast spikes were not followed by large amplitude, long-duration afterhyperpolarizations. Indeed, these spikes presented little evidence of a calcium-activated afterhyperpolarization. A similar low-amplitude, fast spike that fired without triggering a prominent afterhyperpolarization was observed in vivo during antidromic activation of DA neurons (Grace and Bunney, 1983b). This spike was shown to be the initial segment (IS; Coombs et al., 1957) spike component of the DA cell action potential (Grace and Bunney, 1983b). The fast spike component observed here is similar in time course and amplitude to this previously described IS spike. In addition, the fast spike triggered during rebound depolarizations had ap- 
A
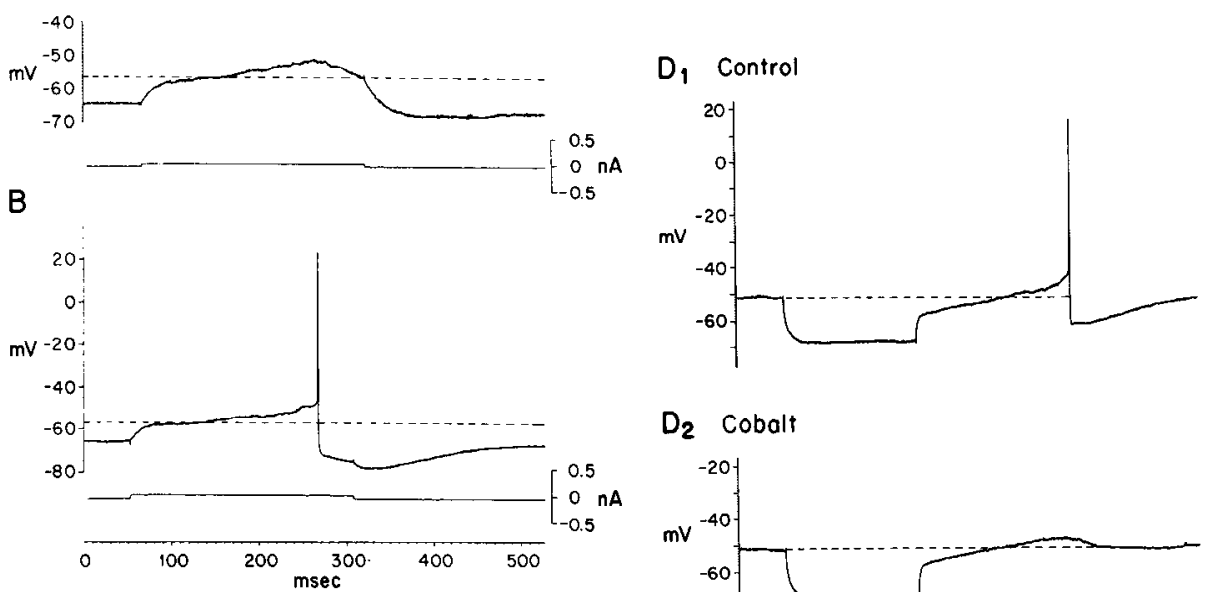

$D_{2}$ Cobalt
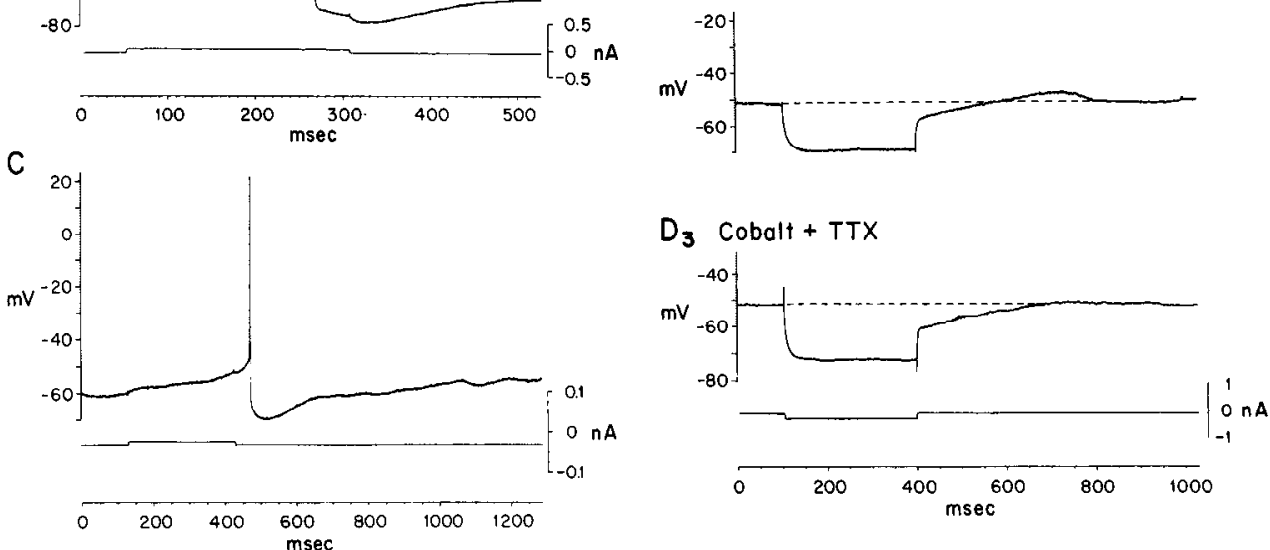

Figure 6. Evidence suggests that the slow depolarization may exhibit voltage-dependent properties. In a hyperpolarized DA neuron, the injection of depolarizing current activates the slow depolarization $(A)$, whereas slightly larger depolarizations trigger an action potential $(B$; dashed lines $=$ depolarization plateau before the slow depolarization). The slow depolarization, once activated, often outlasts the duration of the depolarizing event, being sufficiently regenerative in nature to trigger a spike after the termination of the depolarizing current pulse $(C)$. The slow depolarization can be distinguished from the calcium-dependent rebound depolarization by its sensitivity to blockers. $D_{l}$, Response of a hyperpolarized DA neuron to intracellular injection of a hypcrpolarizing current pulsc. At the offset of current injection, at lcast two membranc cvents occur: (1) a delay in the return of the membrane to its baseline membrane potential (dashed line) and (2) a depolarization of the membrane beyond its resting potential, triggering an action potential. $D_{2}$, Administration of the calcium blocker cobalt ( $\left.2 \mathrm{mM}\right)$ to the Tris-buffered Ringer's solution attenuates the rebound depolarization and prevents activation of spike discharge. $D_{3}$, Administration of the sodium channel blocker TTX (1 $\left.\mu \mathrm{M}\right)$ blocks this remaining rebound depolarization (i.e., the TTX-sensitive slow depolarization). After a transient delay, the DA cell membrane repolarizes to its baseline membrane potential without a concomitant rebound depolarization.

proximately the same amplitude and time course as the notch in the rising phase of the DA neuron action potential.

\section{Cobalt-sensitive membrane potentials}

Low threshold depolarization. A rebound response that transiently depolarizes the DA neuron above its steady-state, baseline membrane potential can be observed following a brief hyperpolarization of the DA neuron membrane or by injecting subthreshold levels of depolarizing current into hyperpolarized DA neurons. This rebound depolarization appears to be composed of more than a single component. The slow depolarization was shown to be one element of this response, since a portion of the resultant depolarization can be attenuated by administering TTX to the slice. However, blockade of the sodiummediated slow depolarization and spike generation by the application of TTX also reveals the presence of a TTX-insensitive low threshold depolarization. As shown above for the TTXsensitive component of the rebound response, this TTX-insensitive depolarization can be triggered either by terminating the hyperpolarization of the DA cell membrane or by directly depolarizing a hyperpolarized DA cell. However, depolarization of a DA cell already in a depolarized state will not activate this
TTX-sensitive depolarization. After selective blockade of the slow depolarization by TTX, the remaining TTX-insensitive depolarizing the DA cell membrane. Increasing the membrane blocker cobalt ( $2 \mathrm{mM}$ ) into the Ringer's (Fig. $8 A$ ).

High threshold calcium spikes. Following TTX administration, only the low threshold depolarization can be activated by depolarizing the DA cell membrane. Increasing the membrane depolarization also failed to trigger other depolarizing membrane processes in TTX-treated DA neurons, even if comparatively large depolarizations were used (Fig. 8B). In contrast, after the application of the potassium channel blocker TEA (2$5 \mathrm{~mm}$ ) to TTX-poisoned slices, one or more large amplitude (75 $\pm 11 \mathrm{mV} ; n=33$; range: $61-85 \mathrm{mV}$ ), long duration $(12.2$ $\pm 3.4 \mathrm{msec}$; range: $7-20 \mathrm{msec} ; n=36$ ), TTX-insensitive spikes could be elicited with only moderate depolarizations (Fig. $8 B_{2}$ ). These spikes were most likely calcium-mediated, since they had a comparatively slow time course with respect to that of the sodium spikes and could be blocked by administering cobalt to the Ringer's ( $1 \mathrm{~mm}$ cobalt: $1 \mathrm{~mm}$ calcium in Tris-buffered Ringer's, Fig. $8 B_{3}$ ). Unlike the low threshold depolarization, depolarization of the DA neuron facilitated current-induced triggering of this spike. Thus, in terms of threshold, duration, amplitude, 

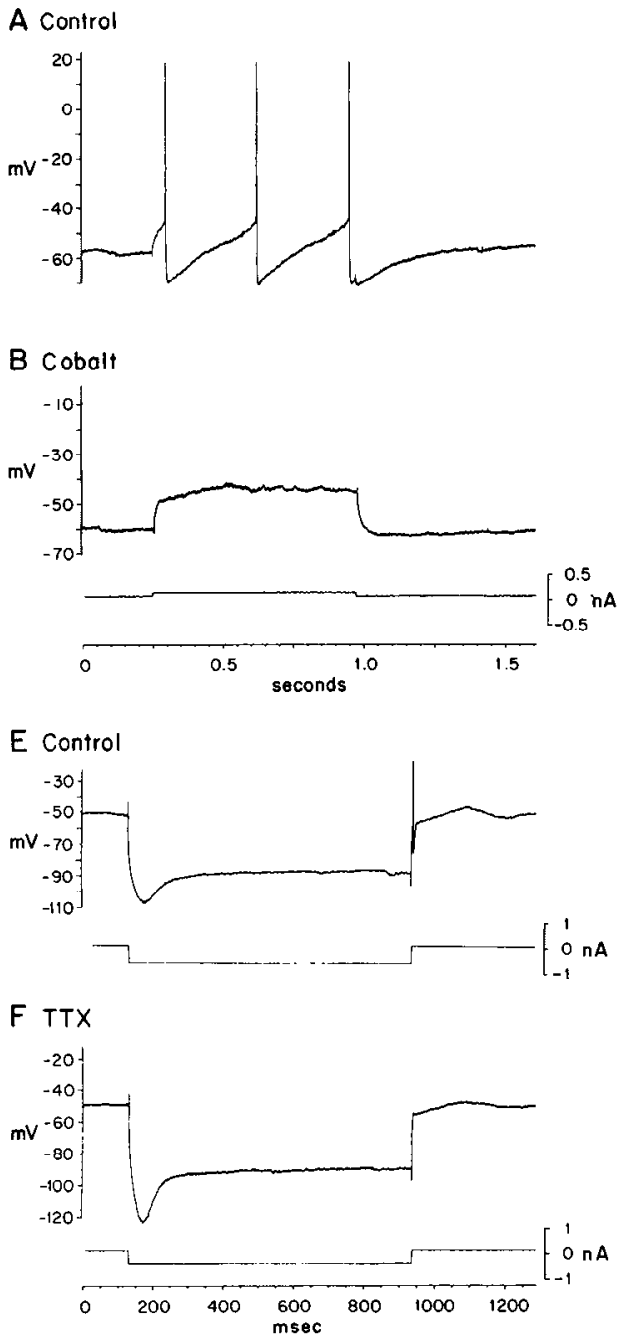
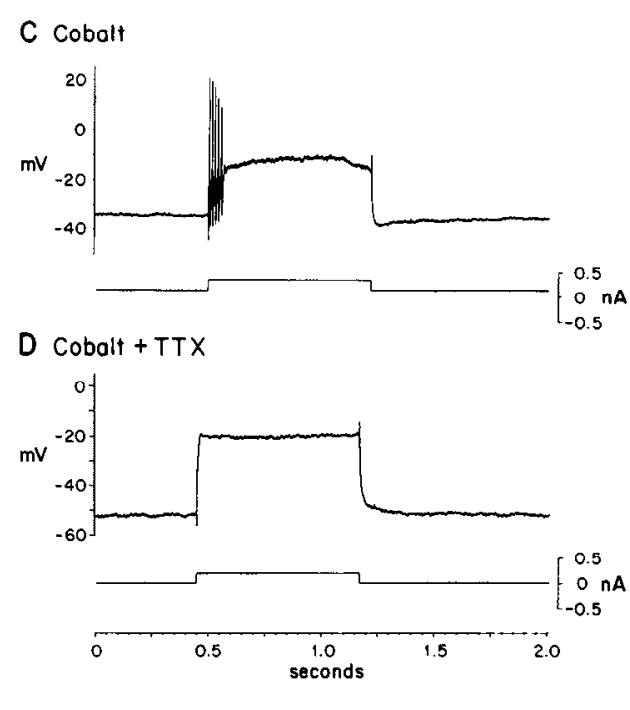

Figure 7. Effects of calcium blockade on action potential generation in DA neurons. $A$, Depolarization of a slowly firing DA neuron triggers the discharge of a series of action potentials. $B$, After cobalt ( $2 \mathrm{mM})$ is added to the Tris-buffered superfusion medium, the depolarization is no longer capable of causing action potential discharge. $C$, Spike firing can be achieved with larger depolarizations. The spikes produced cxhibit high thresholds, are short in duration $(1-1.5 \mathrm{msec})$, and are not followed by afterhyperpolarizations of significant amplitude or duration. The absence of large afterhyperpolarizations following fast spikes allows large membrane depolarizations to trigger a series of these fast spikes in cobalt-treated DA neurons. The spikes are elicited at interspike intervals that are much shorter than those observed for action potential fring in DA neurons in normal Ringer's. $D$, Although this fast spike is not blocked by administcring calcium channel blockers, administration of the sodium blocker TTX $(2-4 \mu \mathrm{M})$ suppresses the discharge of this fast spike, supporting its tentative identification as a sodium-dependent IS spike. $E$, Large membrane hyperpolarizations can elicit a rebound spike showing a similar amplitude and time course without cobalt pretreatment. $F$, The fast rebound spike can be blocked by administering TTX $(2-4 \mu \mathrm{M})$ into the Ringer's. and ionic dependency, these TTX-insensitive spikes were similar to the high threshold spikes (HTS) described in other preparations.

As shown above, HTSs could be triggered after the administration of TEA by depolarizations that previously were incapable of triggering large amplitude, TTX-resistant spikes. TEA administration, therefore, lowered the apparent threshold of the HTS, at least as measured by electrodes located in the soma. Thus, after TEA administration, the HTSs could be triggered by small membrane depolarizations (Fig. $9 A$ ), as well as by the rebound depolarization that occurs following small hyperpolarizations (Fig. 9B). In hyperpolarized DA neurons, membrane depolarizations that were subthreshold for activating the HTS were still capable of triggering the low threshold, cobalt-sensitive depolarization. In contrast to the cobalt-insensitive IS spike reviewed above, the HTSs were followed by large amplitude, long duration afterhyperpolarizations, with both the amplitude and duration of the afterhyperpolarization dependent on the number of HTSs elicited (Fig. 9C).

\section{Nonregenerative membrane potentials}

Long duration (i.e., $>300 \mathrm{msec}$ ) hyperpolarizations of DA neurons revealed the presence of two voltage-dependent conductances: (1) a voltage-dependent sag that occurred during membrane hyperpolarization and exhibited properties similar to those of the anomalous rectifier and (2) a delay in repolarization following a hyperpolarizing current pulse (henceforth referred to as the delayed repolarization). The anomalous rectifier was introduced previously in the section on input resistance to account for the sag. The delayed repolarization occurring during membrane repolarization apparently is inactive at resting potentials but is activated during the transition of the membrane potential from a more hyperpolarized state to a depolarized level, i.e., at the offset of membrane hyperpolarization. This delayed repolarization was observed to slow the repolarization of the membrane potential to baseline levels (Fig. 10B). Although the current underlying this event could not be identified directly without voltage clamping, this event has several properties in common with the A current $\left(I_{A}\right)$ described in other preparations, such as activation during membrane repolarization following a hyperpolarizing pulse, failure to activate at membrane potentials below $-60 \mathrm{mV}$ (Fig. 10A), and an insensitivity to TEA (Fig. 10B), as described by Connor and Stevens (1971). However, in this preparation, administration of the selective potassium blocker 4-AP did not block this putative $I_{A}$ (Fig. 10C), in contrast to the pharmacological specificity of 4-AP in blocking analogous currents in other preparations (Connor and Stevens, 1971; Gustafsson et al., 1982). Cobalt also was ineffective in measurably attenuating this delayed repolarization.

A voltage-dependent delay in membrane repolarization could 
Figure 8. $A$, The low threshold depolarization demonstrates a markedly slower activation and a different sensitivity to ion channel blockers than found for the slow depolarization. $A_{l}$, Continuous hyperpolarization of the DA neuron membrane prevents spontaneous spike discharge in this cell. Injection of a pulse of depolarizing current leads to membrane depolarization and spike firing. $A_{2}$, Administration of the sodium channel blocker TTX $(1 \mu \mathrm{M})$ to the superfusion medium blocks spike firing and reveals a TTX-insensitive component of the membrane depolarization. $-A_{3}$, Subsequent administration of cobalt ( $2 \mathrm{mM}$ ) to the Tris-buffered superfusion medium blocked this low threshold depolarization. Current injection parameters are identical in each case. $B$, In addition to the slower, low threshold cobalt-sensitive depolarizations observed in DA neurons after TTX administration, a type of high threshold, fast calcium spike can also be elicited. $B_{t}$, Increasing levels of depolarization fails to elicit spiking after TTX administration. $B_{2}$, As TEA ( $5 \mathrm{~mm}$ ) is administered to the slice, it initially causes an enhancement of the low threshold depolarization occurring in response to similar levels of membrane depolarization. Over time, this low threshold depolarization increases in amplitude until it triggers an all-ornothing, large amplitude spike. The low threshold depolarization was not replaced by the spike, since the low threshold depolarization can still be triggered alone with smaller depolarizing stimuli. $B_{3}$, Subsequent application of cobalt to the Tris-buffered Ringer's ( $2 \mathrm{mM})$ blocks activation of the high threshold, TTX-insensitive spike.
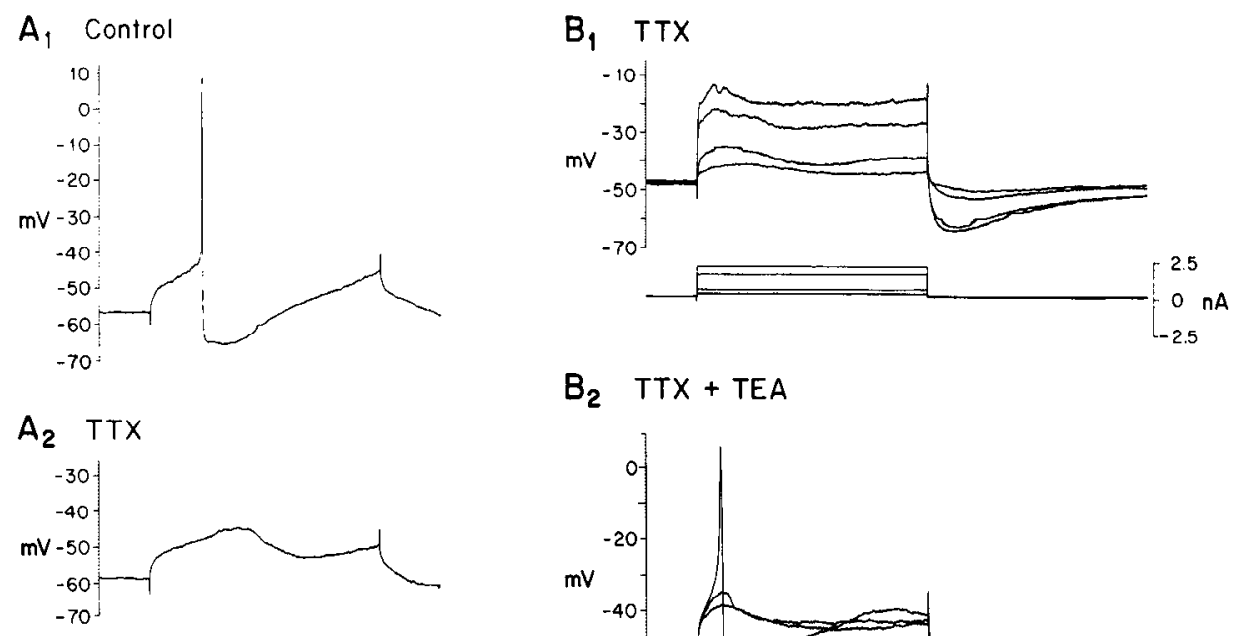

$B_{2}$ TTX + TEA

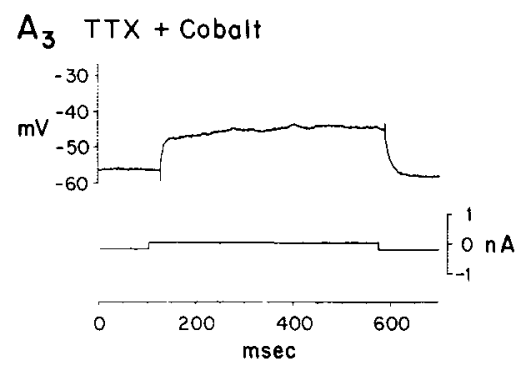

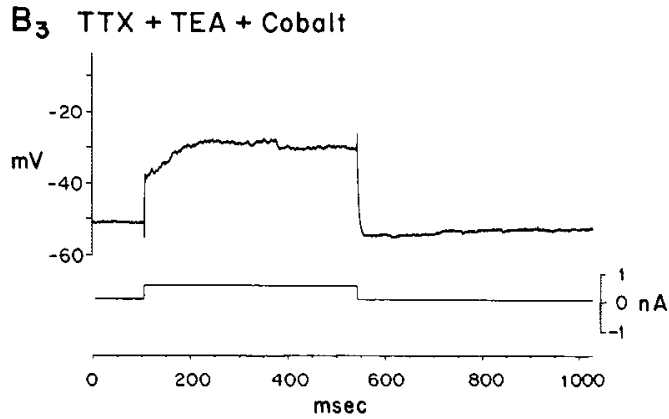

act to modulate other rebound events, such as the rebound depolarizations. In this preparation, hyperpolarizations sufficient to activate this delayed repolarization cause an attenuation of the rebound depolarization (Fig. 11 $A$ ) described earlier in this paper. The degree of attenuation produced appears to depend on the amount of membrane hyperpolarization induced. Thus, although a small hyperpolarization of the DA neuron will trigger a rebound membrane depolarization and spike firing, increasing levels of hyperpolarization will actually prevent this rebound excitation at the same time that it activates the delayed repolarization (Fig. 11B).

\section{Discussion}

Identifying the factors that regulate DA neuron activity and how this regulation is affected by drug administration may yield information of relevance to the etiology and treatment of some neurological and psychiatric disorders. However, with respect to applying the data gathered from recordings of neuronal activity to the analysis of drug action, it is first necessary to establish that the neurons recorded are indeed of the neurochemical subtype one wishes to investigate.

\section{Neuron identification}

Based on electrophysiological criteria, 2 general classes of neurons could be distinguished in the zona compacta region of the substantia nigra and in the ventral tegmental area. The principal ncuron encountcred was typically spontaneously active and fired in a pacemaker pattern. The long duration, high threshold action potentials were preceded by a slow depolarization and followed by a prominent afterhyperpolarization. Labeled neurons of this type had medium-sized fusiform or multipolar somata that gave rise to a small number of sparsely branching dendrites. These neurons consistently demonstrated double labeling for tyrosine hydroxylase-like immunoreactivity, indicating that they were of the dopaminergic subtype. A second neuron type rarely exhibited spontaneous activity and fired short duration $(<1.5 \mathrm{msec})$ action potentials in a repetitive manner when depolarized from resting potentials but would fire high frequency $(3-5 \mathrm{msec}$ interspike interval) bursts of spikes when depolarized from hyperpolarized membrane potentials. Labeled neurons exhibiting these electrophysiological properties had large somata ( $>30 \mu \mathrm{m}$ diameter), with numerous highly branched dendritic processes 
A
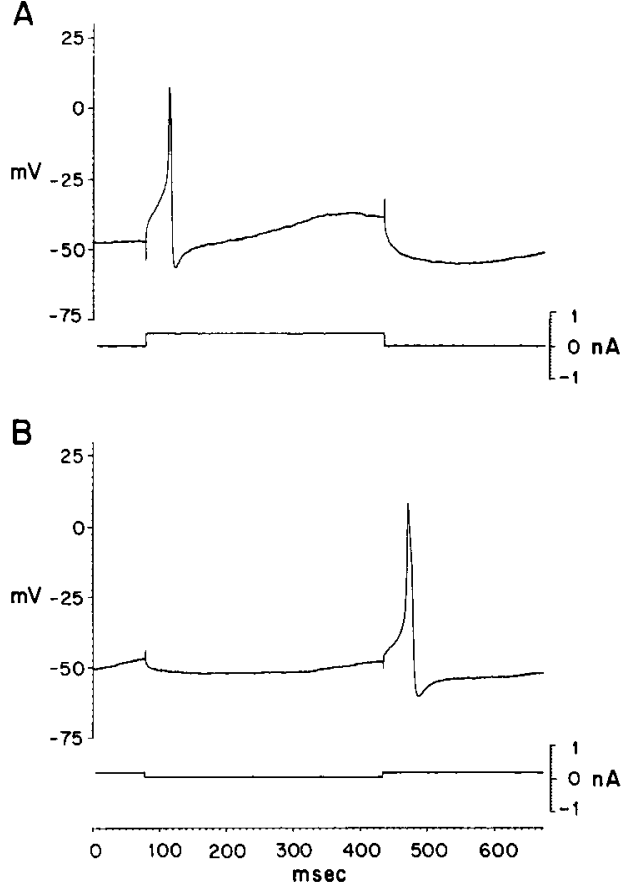

$c_{1}$

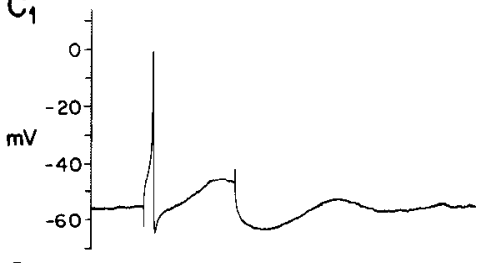

$\mathrm{C}_{2}$

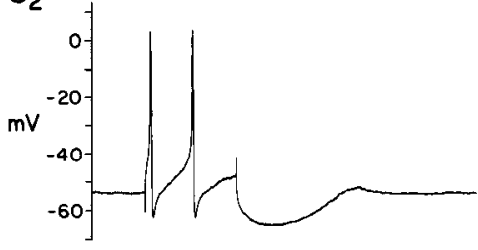

$\mathrm{C}_{3}$

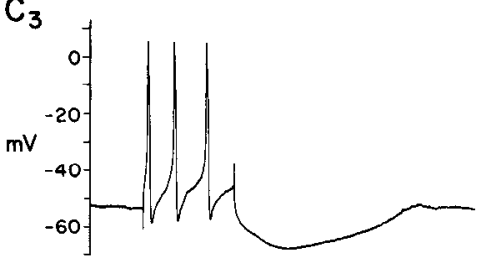

$\mathrm{C}_{4}$

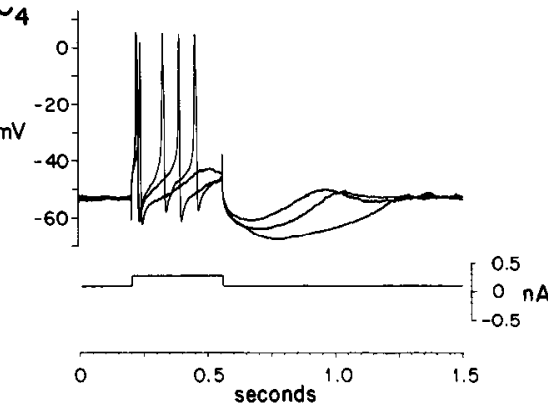

Figure 9. The TTX-insensitive spike cannot be triggered in TTX-treated DA neurons without administering TEA. However, after treatment with TEA, this spike can be triggered easily by small membrane potential changes. $A$, Following treatment with TTX and TEA, depolarization of a depolarized DA neuron triggers a calcium spike. $B$, This spike also can be triggered by the rebound depolarization occurring at the offset of a brief hyperpolarization of the membrane of depolarized DA neurons. $C$, The TTX-insensitive spikes are followed by prominent afterhyperpolarizations. In this case, equal amplitude depolarizations of a DA neuron treated with TTX and TEA triggers 1, 2, or 3 spikes $\left(C_{1}-C_{3}\right)$. Overlaying these 3 traces shows that the amplitude and duration of the afterhyperpolarizations following the current pulses are dependent on the number of spikes elicited, with more spikes associated with more prominent afterhyperpolarizations. $\left(C_{4}\right.$, overlay of traces $C_{1}-C_{3}$.)
A

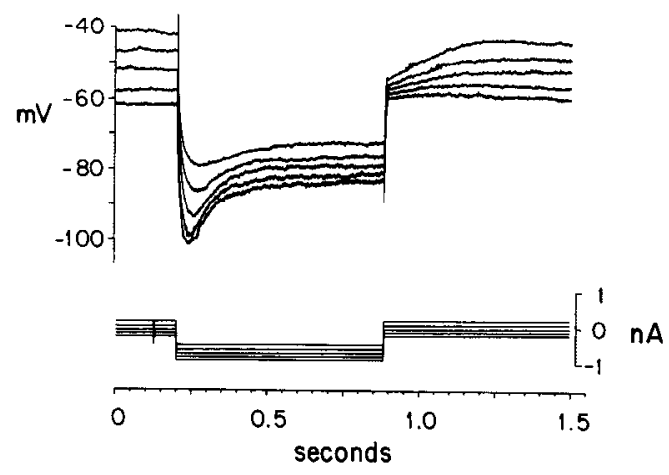

B Control

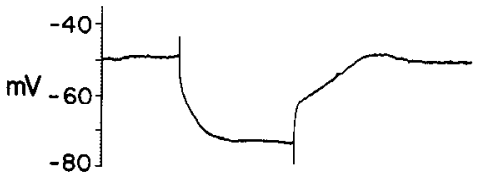

C 4-AP

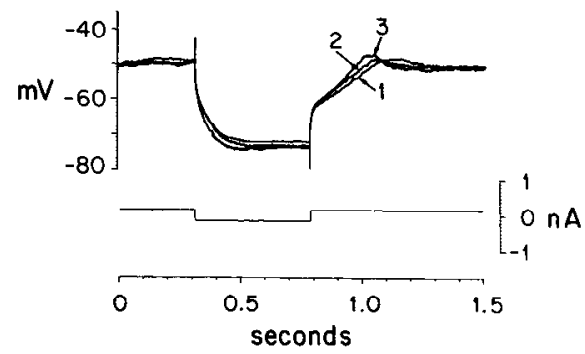

Figure 10. A, Both the anomalous rectifier and the delayed repolarization exhibit voltage-dependent properties. In a DA neuron showing low levels of spontaneous activity, depolarization decreased and hyperpolarization increased the time-dependent "sag" in the membrane potential, which is reported to reflect the magnitude of the anomalous rectification. Hyperpolarization of the membrane below about - $60 \mathrm{mV}$ also prevented activation of the delayed repolarization. $B, C$, Although the delayed repolarization induced in DA neurons during repolarization from hyperpolarized potentials shows a time course and voltage dependence similar to that described for $I_{A}$-type currents, it is not blocked to a significant extent by 4-AP. Thus, application of 4-AP to a TEA- and TTX-treated DA neuron is capable of blocking only a portion of this delayed repolarization. $B$, Control response, TTX $=1 \mu \mathrm{M} ;$ TEA $=4 \mathrm{mM}, C$, Overlay of control response (1) with response obtained after addition of 4-AP. Concentrations: $5 \mathrm{~mm}(2)$ and $20 \mathrm{~mm}(3)$. 


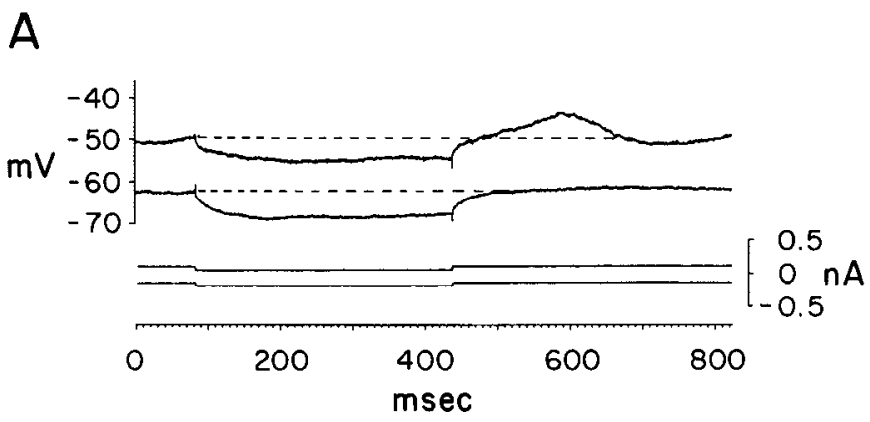

B

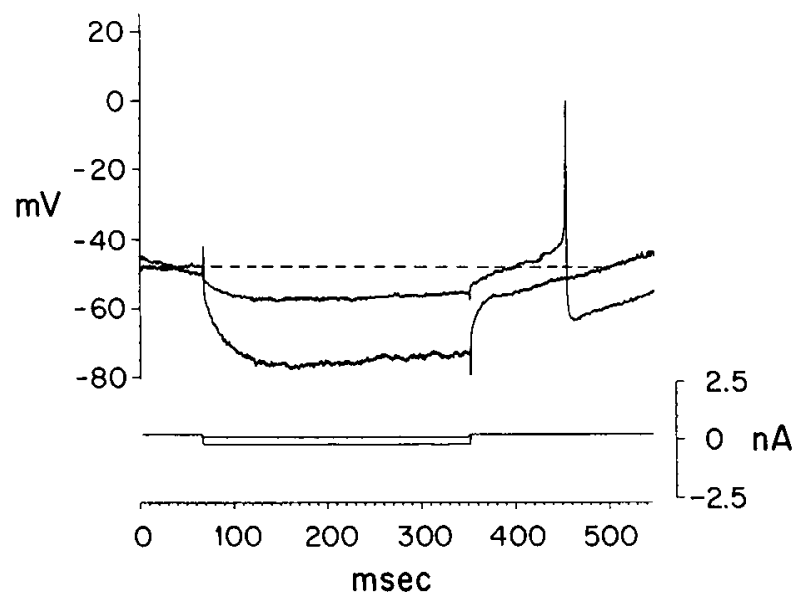

Figure 11. A, The amplitude of the rebound depolarization in DA neurons is strongly influenced by the baseline membrane potential. Intracellular injection of a hyperpolarizing current pulse into a slightly depolarized but nonfiring DA neuron (upper trace) triggers a rebound dcpolarization at the offset of the pulse. However, injection of a pulse of current of the same amplitude into a hyperpolarized DA neuron (lower trace) does not trigger a rebound depolarizing response. $B$, In DA neurons, larger membrane hyperpolarizations produce comparatively smaller rebound depolarizations. Thus, hyperpolarization often triggers rebound spiking in nonfiring DA neurons (upper trace). In contrast, larger hyperpolarizations prevent rebound activation of the low threshold depolarization, presumably because of the concurrent activation of the delayed repolarization (lower trace). (Dashed lines $=$ baseline membrane potential.)

emanating from the soma, some of which were observed to extend dorsally into the reticular formation. Neurons of this type did not demonstrate tyrosine hydroxylase immunoreactivity.

Drawing from the range of critcria used to identify DA neurons in vivo, this second neuronal type had sufficiently different morphological and physiological characteristics to allow it to be readily distinguished from DA neurons. Thus, in addition to the inability to label this neuron immunocytochemically, this latter neuronal type resembled the morphological characteristics of neurons that had been classified as non-dopaminergic based on in vivo (Tepper et al., 1987: non-DA neurons) and in vitro (Nakanishi et al., 1987: zona reticulata neurons) recordings and staining of neurons in this brain region. Furthermore, the firing pattern observed for this class of neurons is consistent with the electrophysiological characteristics reported by others for neurons in this brain region (Llinás et al., 1984; Nedergaard et al., 1988a, b; Greenfield et al., 1988), which some have identified as non-dopaminergic zona reticulata neurons (Nakanishi et al., 1987).

\section{DA neuron morphology}

Consistent with the morphology reported for physiologically identified DA neurons labeled during in vivo intracellular recordings (Grace and Bunney, 1983b; Tepper et al., 1987) and with Golgi (Schwyn and Fox, 1974; Juraska et al., 1977) fluorescence histochemical (Lindvall and Björklund, 1974, 1978), and immunocytochcmical (Hökfelt et al., 1976) studies of midbrain DA neurons, the identified DA neurons labeled here could be divided into 3 morphological classes: (1) multipolar substantia nigra cells, (2) bipolar substantia nigra cells, and (3) ventral tegmental area neurons that ranged from multipolar to bipolar in shape. The DA neurons exhibited 3-6 thick, sparsely branching major dendrites emanating from the soma, with the dendrites typically oriented in a mediolateral direction and remaining within the the dorsal-ventral confines of the substantia nigra zona compacta or extending ventrally into the zona reticulata (Juraska et al., 1977; Preston et al., 1981; Grace and Bunney, 1983b; Tepper et al., 1987; Grace, 1987, 1988). The dendrites of the bipolar substantia nigra neurons typically remained within the confines of the zona compacta, which is consistent with the morphology described for the dorsal tier DA neurons (Fallon et al., 1978; Gerfen et al., 1987a, b) and the acetylcholinesterase-rich DA neurons identified by Jiminez-Castellanos and Graybiel in primates (1987). The more ventral multipolar DA neurons of the substantia nigra also had dendrites confined to the zona compacta but, in addition, had 1-3 dendrites that descended into the zona reticulata. This morphology is similar to that of the ventral tier DA neurons described by others (Björklund and Lindvall, 1975; Gerfen et al., 1987a, b). Based on this dendritic branching pattern, the more dorsal fusiform DA neurons would be restricted to sampling synaptic input confined to the zona compacta region, whereas the more ventral multipolar neurons also have dendrites that could receive afferent inputs terminating in the zona reticulata as well. DA neuron dendrites were found to exhibit spinule-like protrusions at irregular intervals (Juraska et al., 1977; Preston et al., 1981; Tepper et al., 1987; Grace, 1987, 1988), with the dendrites ending in forks or tufts (Preston et al., 1981; Grace and Bunney, 1983b). These terminal dendritic forks consisted of 2-3 fine processes that appeared to "wrap around" an element in the zona reticulata or in the zona compacta. Indeed, this morphological specialization is not dissimilar from that described for the basket-like terminations of axons around the base of pyramidal neurons, as described in the cerebral cortex (Jones, 1975). One speculative hypothesis to account for this type of morphological arrangement would be that the terminal tufts are dendritic release sites for DA acting on non-dopaminergic nigral neurons, such as the DA-sensitive nigrothalamic neurons reported by others (Ruffieux and Schultz, 1980; Waszczak and Walters, 1983).

\section{Physiological properties of $D A$ neurons}

Despite the presence of 3 morphological subtypes of DA neurons, all identified DA neurons exhibited similar electrophysiological characteristics. Furthermore, DA neurons recorded in vitro displayed many of the same physiological characteristics reported for in vivo intracellular recordings from this class of cells, such as (1) a long duration action potential, (2) a slow, depolarizing pacemaker potential, (3) a high spike threshold, and (4) a prominent afterhyperpolarization (cf. Grace, 1987). However, 2 striking differences were found in the membrane properties of DA neurons recorded in the in vitro preparation 
when compared to those reported in vivo. These differences relate to DA cell input resistance and firing pattern. DA cells recorded in vivo typically exhibit input resistances averaging about $30 \mathrm{M} \Omega$ (Grace and Bunney, 1983a). In contrast, DA cells recorded in vitro have input resistances 3-6 times this value; i.e., averaging about $170 \mathrm{M} \Omega$, which is consistent with that reported by others using in vitro recordings from midbrain slices (Pinnock, 1984; Kita et al., 1986; Lacey et al., 1987; Silva and Bunney, 1988) and dissociated cell cultures (Chiodo and Kapatos, 1987; Chiodo, 1988; Ort et al., 1988). Although it is possible that this may represent a difference in the stability of the electrode penetration between these two preparations, this explanation appears to be inadequate to account completely for this disparity, since (1) DA neurons recorded intracellularly in vivo exhibited action potential durations, firing rates, and firing patterns similar to those observed extracellularly in vivo where penetration injury should not be a factor, and (2) penetrations of spontaneously firing DA neurons in vivo could be maintained for periods of up to $2.5 \mathrm{hr}$ without significant increases in input resistance (Grace, 1987). The observation that DA neurons recorded in vivo are under constant bombardment with chloridedependent i.p.s.p.s (Grace and Bunney, 1985), which were not observed to an equivalent extent in the present study, may partially account for this difference in input resistance. Indeed, Lacey et al. (1988) reported that application of GABA to substantia nigra zona compacta neurons in vitro will reduce their input resistance by $80 \%$ with respect to controls.

\section{Firing pattern}

A second major difference in the physiology of DA neurons recorded in vitro versus those reported in vivo is in the firing pattern observed. It is estimated that about $50-70 \%$ of the DA neurons in the substantia nigra of anesthetized rats are spontaneously active (Grace and Bunney, 1986; Grace, 1987). The spontaneously active DA neurons recorded in vivo fired in 2 distinguishable patterns: (1) a single spiking pattern characterized by individual spikes or doublets occurring with irregular interspike intervals and (2) burst firing, which consists of trains of 3-8 spikes of decreasing amplitude and increasing duration occurring with comparatively short interspike intervals (i.e., 50$150 \mathrm{msec}$; average: $73 \pm 13 \mathrm{msec}$ ) but with prolonged periods of postburst inhibition of activity (approximately $200-450 \mathrm{msec}$; Grace and Bunney, 1984a, b). In contrast, identified DA neurons recorded in vitro fired exclusively in a very regular, pacemakerlike pattern, somewhat similar to that found in hypothalamic histaminergic neurons recorded in vitro (Haas and Reiner, 1988). Furthermore, depolarization of the DA cell increased the frequency of spike discharge without altering this pacemaker firing pattern. DA neuron depolarization in vivo typically triggered a burst firing pattern (Grace and Bunney, 1984b). In our hands, burst firing could not be induced in identified DA neurons in vitro by any of the wide range of manipulations of membrane potential or time course of current injections examined in this study. It is unclear why DA neurons in vitro should display such markedly different patterns of discharge when compared with those recorded in vivo. One possibility is the lack of GABAergic bombardment (Grace and Bunney, 1985) and the consequent high input resistance found in the in vitro preparation, which could serve to lock the discharge pattern into a very regular structure controlled primarily by the slow depolarization and the afterhyperpolarization. This type of very regular discharge pattern is rarely observed in DA neurons recorded in vivo, al- though intracellular injection of the calcium chelator EGTA into DA neurons in vivo will cause them to fire in a very similar, highly regular pacemaker pattern (Grace and Bunney, 1984a) as well as prevent membrane depolarization from eliciting burst firing (Grace and Bunney, 1984b). The relevance of these observations to the data discussed here, however, is not clear at present.

\section{Anomalous rectification}

Intracellular injection of hyperpolarizing current pulses elicited 2 events that may play a role in regulating DA cell activity. Hyperpolarization of $\mathrm{DA}$ neurons to $-75 \mathrm{mV}$ or more was accompanied by the development of a prominent sag in the membrane potential (Grace and Bunney, 1983a; Pinnock, 1985; Kita et al., 1986), thereby decreasing the amount of hyperpolarization produced. The change in conductance underlying this sag has properties similar to those described for the anomalous rectifier in cortical neurons (Schwindt et al., 1988) and appears to be larger in amplitude than that observed for DA neurons recorded in vivo (Grace and Bunney, 1983a). The anomalous rectifier is thought to play a role in maintaining pacemaker firing patterns of neurons (Crepel and Penit-Soria, 1986) and thus may contribute to the pacemaker firing pattern observed here. An anomalous rectifier producing a similar, highly prominent sag in the membrane potential during hyperpolarization has been reported in neocortical pyramidal cells (Schwindt et al., 1988 ), with this sag in the membrane voltage described as the "hallmark" of a slow anomalous rectifier. This sag apparently is composed of an instantaneous component and a time-dependent component, similar to that described by Yarom and Llinás (1987) in the inferior olive, since both the initial and the steadystate current-voltage relationships exhibit increasing deviations from linearity with larger degrees of hyperpolarization. The anomalous rectification was blocked in DA neurons in vivo by intracellular injection of TEA (Grace and Bunney, 1984a). The function of the anomalous rectifier in DA cell firing is unclear, but one possibility is that the action of this conductance may be dependent on the membrane potential. Thus, it should function to decrease excitability in very hyperpolarized DA neurons by increasing membrane conductance, while also serving to maintain the pacemaker-like activity in spontaneously firing DA neurons by keeping the membrane potential positive to the potassium equilibrium potential (Crepel and Penit-Soria, 1986; Schwindt et al., 1988) and possibly closer to the threshold for activating the slow depolarization.

\section{Delayed repolarization}

In addition to the anomalous rectifier, hyperpolarizing pulses revealed the presence of a conductance change that caused a delayed repolarization of the membrane potential. Although the precise nature of this event cannot be demonstrated conclusively without voltage clamping, one interpretation of the results suggests that this delayed repolarization may be caused by an A-type potassium current $\left(I_{A}\right)$. This delayed repolarization exhibits properties that are similar to those produced by the well-defined $I_{A}$ in other preparations with respect to (1) its activation during membrane repolarization from hyperpolarized membrane potentials, (2) its lack of activation at membrane potentials more negative than $-60 \mathrm{mV}$, and (3) its insensitivity to TEA (10 mM; Connor and Stevens, 1971; Neher, 1971; Thompson, 1977; Gustafsson et al., 1982). On the other hand, the insensitivity of this event to blockade by 4-AP stands in contrast to the reported 
pharmacological selectivity of 4- $\mathrm{AP}$ for $\mathrm{I}_{\mathrm{A}}$ channel blockade (Connor and Stevens, 1971; Gustafsson et al., 1982). However, a 4-AP-insensitive delayed repolarization with properties otherwise similar to the $I_{A}$ has been reported in at least one other vertebrate neuronal type (hypothalamic histamine-containing neurons; Haas and Reiner, 1988). This distinction may be particularly relevant for DA neurons, since 4-AP appears to block a functionally different membrane event that is involved in regulating DA cell excitability and spike threshold (Grace and Onn, 1988a)

As reviewed, the putative $I_{A}$ should be triggered following hyperpolarizing events under conditions that elicit the rebound depolarization. Indeed, coactivation of a putative $I_{A}$ may be one reason that larger hyperpolarizing current pulses do not trigger rebound bursts of spikes in DA neurons, since the proposed conductance increase could shunt the rebound depolarizing events. This stands in contrast to the rebound low threshold spike (LTS) and burst firing produced at the offset of large membrane hyperpolarizations in some other types of neurons (e.g., Llinás and Yarom, 1981a, b; Grace and Llinás, 1984; Jahnsen and Llinás, 1984a, b; Nakanishi et al., 1987; Nedergaard et al., $1988 \mathrm{a}, \mathrm{b})$. This interdependence of the $\mathrm{I}_{\mathrm{A}}$ and the LTS was suggested by Bossu et al. (1985) to account for the responses of sensory neurons to membrane hyperpolarization. In that paper, the authors concluded that the excitability of neurons could be more strongly influenced by either the low threshold, transient calcium current (Nowycky et al., 1985) or the $I_{A}$, depending on the relative level of activation of these currents (i.e., which is more prominent) at the membrane potential examined. Drawing from this model, the spike afterhyperpolarization may be of sufficient amplitude to activate a rebound low threshold depolarization, slow depolarization, and spike in depolarized DA cells, whereas stronger hyperpolarizing stimuli should actually diminish rebound excitation by preferential activation of the $I_{A}$. Indeed, the voltage-dependent properties of the putative $I_{A}$ could explain why the low threshold depolarizations observed here are not all-or-nothing events, as reported for the LTS in other preparations (Llinás and Yarom, 1981a, b), but instead show graded amplitudes that vary with the membrane potential. In this model, it is possible that the graded amplitude of an allor-nothing LTS could arise from variations in the level of activation of the putative $I_{A}$.

\section{DA neuron spike subcomponents}

The slow depolarization drives the membrane of the DA neuron to rather depolarized potentials to trigger spike firing. This inordinately high spike threshold is also a distinctive feature of identified DA neurons recorded in vivo (Grace and Bunney, 1984a). The action potential exhibits an inflection in the rising phase of the spike waveform due to the delay between activation of the IS spike and triggering of the somatodendritic (SD) spike, although it is not as prominent as that observed in vivo (Grace and Bunney, 1983b). A shortened IS-SD delay is indicative of a higher safety factor for somatodendritic invasion of the IS spike (Renshaw, 1942; Brock et al., 1953), which in this case may be attributed to the higher membrane resistance of DA neurons in vitro that should facilitate current spread into the dendrites. The IS spike-generating zone is the lowest threshold spiking region of vertebrate neurons (Coombs et al., 1957). As the lowest threshold component of the action potential, the variability of DA cell spike thresholds at different membrane potentials (Grace and Bunney, 1983b; Grace, 1987) is likely to reflect changes in the threshold of the IS spike. This variability may be due to the comparatively distal location of the IS region with respect to the DA cell soma (Juraska et al., 1977; Preston et al., 1981; Grace and Bunney, 1983a, b; Tepper et al., 1987). This is consistent with previous in vivo studies in which an electrotonically distal location for the IS spike was inferred by comparing intracellular and extracellular action potentials (Grace and Bunney, 1983b). The absence of a prominent afterhyperpolarization following the IS spike combined with the sensitivity of this spike to TTX application is consistent with this being a sodium-mediated spike.

Blockade of the IS spike and slow depolarization with TTX reveal 2 calcium-dependent depolarizations that are involved in DA neuron spike generation: the low threshold depolarization and the HTS. The low threshold depolarization in DA neurons, although similar in many respects (e.g., time course, de-inactivation with hyperpolarization, sensitivity to cobalt) to the LTS described in other vertebrate neurons (Llinás and Yarom, 1981a, b, 1986; Jahnsen and Llinás, 1984a, b; Llinás et al., 1984; Grace and Llinás, 1984), nonetheless shows some unique properties in this preparation. Thus, although the rebound LTS in many vertebrate neurons is mediated primarily if not exclusively by calcium, the rebound depolarization in DA neurons appears to be composed of both a calcium-dependent low threshold depolarization and a sodium-mediated slow depolarization. The LTS has been shown in many other vertebrate neurons to be important in the regulation of cell excitability. Thus, very hyperpolarized neurons were found to emit proportionately larger amplitude LTSs than could be produced in less hyperpolarized neurons (Llinás and Yarom, 1981a, b; Jahnsen and Llinás, 1984a, b; Grace and Llinás, 1984; Wilcox et al., 1988). However, in DA neurons, the opposite appears to be the case; i.e., whereas smaller hyperpolarizations elicit rebound depolarizations in DA neurons at resting or at depolarized membrane potentials, rebound depolarizations could not be elicited in more hyperpolarized DA neurons. Furthermore, larger hyperpolarizations will actually block activation of the rebound depolarization regardless of the initial membrane potential. This blockade could be due to a depolarization-induced activation of the putative $I_{A}$. By increasing the membrane conductance during membrane repolarization, activation of the $I_{A}$ could serve to shunt the currents mediating the rebound depolarization. Thus, the membrane potentials at which the putative LTS can be activated in DA cells is more highly constrained than has been found in other neurons. As a consequence, although the low threshold depolarization could support spontaneous spiking in DA neurons when it is activated following the spike afterhyperpolarization, it probably would play less of a role in a tonically hyperpolarized DA neuron due to activation of the putative $I_{A}$.

A second calcium-dependent depolarization observed in DA neurons is similar to that described as the HTS in other preparations (Schwartzkroin and Slawski, 1977; Wong et al., 1979; Llinás and Yarom, 1981a, b; Grace and Llinás, 1984; Kita et al., 1986; Ort et al., 1988). In contrast to the low threshold depolarization, depolarization of TTX-treated DA neurons will not activate HTSs. The HTS can, however, be triggered by comparatively small depolarizations after pretreatment of DA neurons with the potassium blocker TEA. The HTS is nonetheless apparently distinct from the low threshold depolarization, since the low threshold depolarization can still be elicited after TEA using smaller depolarizing pulses. The ability of TEA to reveal the HTS could be accounted for by several mecha- 
nisms; for example, it could block a local resting potassium conductance or delayed rectifier, either of which could conceivably prevent activation of a nearby spiking zone. However, given (1) the high baseline input resistance of the DA neurons, (2) the lack of significant alterations in input resistance or current-induced depolarization of the soma by TEA (Fig. 8, $B_{1}-$ $\left.B_{3}\right)$, (3) the large degree to which the soma can be depolarized by current injection in the absence of TEA (Figs. $7 D, 8 B_{1}$ ), and (4) the extent to which TEA lowers spike threshold, the evidence appears to favor an action of TEA on HTS spike threshold similar to that proposed by Llinás et al. (1984), i.e., that the blockade of membrane potassium channels by TEA facilitates intraneuronal electrotonic current spread into more distal spikegenerating zones. TEA administration would thereby cause spike generating zones located in distal dendrites to become comparatively more depolarized in response to a given amount of somatic depolarization, causing lowering of the apparent threshold of the HTS spike as measured at the soma. Indeed, when one considers that the HTS cannot be activated by large depolarizations of the somata of DA neurons despite their high input resistance, the requirement for TEA to elicit HTSs could indicate that the HTS spike-generating region is located at a significant distance from the soma. One possible location could be the distal fork-like dendritic tufts described previously.

In contrast to its effects on HTS firing, TEA administration only produces an increase in the amplitude of the low threshold depolarization without changing its threshold for activation. Although not conclusive, this observation, plus the observation that it can be elicited by small amplitude depolarizations of the soma, suggests that the low threshold depolarization is generated at an electrotonically proximal site with respect to the soma and is distinct from the HTS. This may also be true for the slow depolarization, since (1) it can also be activated by brief depolarizations of the soma, and (2) its rate of activation is strongly dependent on the membrane potential of the soma. If, on the other hand, these processes were mediated at more electrotonically distal sites, one may predict that longer potential changes or larger currents would be required for their activation.

The HTSs are likely to comprise the majority of the SD spike, since (1) both the SD spike and the HTS can be blocked by cobalt administration, and (2) TEA was found to increase the duration of the SD and the HTS without affecting the IS spike duration (unpublished observations). During normal spike activity, the IS spike must be of sufficient amplitude and optimally located to trigger the HTSs. Furthermore, the DA neuron itself must be adequately depolarized to prevent shunting of the IS spike-induced depolarizing current away from the dendrites. Depolarization of the DA cell soma mediated by the slow depolarization and the low threshold depolarization would thereby facilitate depolarization of the dendrites and triggering of the HTS. Thus, antidromic activation (Grace and Bunney, 1983b) and the rebound from large hyperpolarizations (Fig. $7 E$ ) can trigger the IS spike without activating the SD spike, presumably because the soma and proximal dendrites are insufficiently depolarized (due to the absence of either a preceding spontaneous slow depolarization or rebound low threshold depolarization) to facilitate the spread of the IS spike into the dendrites. Firing of the calcium-mediated HTS would give rise to the spike afterhyperpolarization, which is mediated by a calcium-activated potassium conductance in DA neurons (Grace and Bunney, 1984a). In this way, triggering the HTS would cause a rapid and simultaneous repolarization of the extensive dendritic tree of
DA neurons, thereby resetting the membrane potential and delaying the initiation of a subsequent slow depolarization and low threshold depolarization. Blockade of the HTS by cobalt or triggering of the IS spike by antidromic activation allows the isolated IS spike to fire at much higher frequencies than could normally be attained by a DA neuron firing full amplitude ISSD action potentials due, in part, to the absence of this concomitant long-duration afterhyperpolarization following these sodium-mediated IS spikes (e.g., Fig. 7C).

\section{Spike generation in DA neurons}

Although speculative in nature, the results provide sufficient evidence to construct a preliminary model of spontaneous action potential generation in DA neurons. In this model, the spontaneous slow depolarization, originating near the soma, slowly depolarizes the DA neuron membrane until the IS region reaches spike threshold. The IS spike then spreads across the soma and proximal dendrites (already depolarized by the slow depolarization or low threshold depolarization) to trigger the more distally located dendritic HTS generation sites. The calciummediated HTSs then trigger a calcium-activated afterhyperpolarization that causes widespread hyperpolarization of the soma and the expansive dendritic tree. The rebound from this afterhyperpolarization could then trigger a rebound low threshold depolarization, which would in turn activate the slow depolarization and reinitiate the spike generation sequence. In the absence of afferent modulatory activity, this sequence of events, coupled with their modulation by the prominent anomalous rectifier, could underlie the pacemaker-like spontaneous activity pattern recorded in DA neurons in vitro. Indeed, potentiation of the effects of these depolarizations by the comparatively high input resistance could serve to lock the DA neuron into this nonvarying pacemaker-like firing pattern. However, the factors responsible for modulating this pacemaker pattern into the more typical irregular and burst firing patterns recorded from DA neurons in vivo are unknown at present.

\section{Summary}

Despite the differences in the electrophysiological properties of DA neurons recorded in vivo versus those observed in vitro, DA neurons can nonetheless be identified in each preparation by certain characteristic electrophysiological properties, such as the presence of the slow depolarization, a slow firing rate, a long spike duration, and a high threshold for action potential discharge. The slow depolarization appears to be responsible for maintaining spontaneous spike discharge in DA neurons even in the absence of long-loop afferents and may be a factor in allowing transplanted DA neurons to retain their intrinsic spikegenerating activity.

The in vitro preparation enhances one's ability to identify the endogenous membrane properties of a neuron versus those arising from the interaction of the neuron with the brain system in situ. Thus, although burst firing can be triggered in DA neurons in vivo by depolarization (Grace and Bunney, 1984b), recordings in the in vitro preparation suggest that a factor that "enables" the burst firing mode has been removed. If so, this preparation would facilitate inquiries into the mechanism of DA neuron burst firing. Furthermore, the presence of DA neurons that do not exhibit spontaneous activity in the in vitro preparation provides evidence that the state of DA neuron activity may be regulated by factors other than the striatonigral pathway (Grace and Bunney, 1986; Grace, 1987). Comparisons of cellular prop- 
erties across preparations may thereby provide direction to efforts focused at understanding and eventually modifying the activity of DA neurons in the behaving organism.

\section{References}

Adrian, R. H. (1969) Rectification in muscle membrane. Prog. Biophys. Mol. Biol. 19: 340-369.

Bargas, J., E. Galarraga, H. T. Chang, and S. T. Kitai (1988) Electrophysiological and double-labeling immunohistochemical analyses of neurons in the substantia nigra zona compacta of the rat. Soc. Neurosci. Abstr. 14: 1025.

Björklund, A., and O. Lindvall (1975) Dopamine in dendrites of substantia nigra neurons: Suggestions for a role in dendritic terminals. Brain Res. 83: 531-537.

Bossu, J.-L., J.-L. DuPont, and A. Feltz (1985) $\mathrm{I}_{\mathrm{A}}$ current compared to low threshold calcium current in cranial sensory neurons. Neurosci. Lett. 62: 249-254.

Brock, L. G., J. S. Coombs, and J. C. Eccles (1953) Intracellular recording from antidromically activated motoneurones. J. Physiol. (Lond) 122: 429-461.

Brodie, M. S., and T. V. Dunwiddie (1987) Cholecystokinin potentiates dopamine inhibition of mesencephalic dopamine neurons in vitro. Brain Res. 425: 106-113.

Bunney, B. S., and G. K. Aghajanian (1976) D-Amphetamine-induced inhibition of central dopaminergic neurons: Mediation by a striatonigral feedback pathway. Science 192: 391-393.

Bunney, B. S., and A. A. Grace (1978) Acute and chronic haloperidol treatment: Comparison of effects on nigral dopaminergic cell activity. Life Sci. 23: 1715-1728.

Bunney, B. S., J. R. Walters, R. H. Roth, and G. K. Aghajanian (1973) Dopaminergic neurons: Effect of antipsychotic drugs and amphetamine on single cell activity. J. Pharmacol. Exp. Ther. 185: 560-571.

Chéramy, A., V. Leviel, and J. Glowinski (1981) Dendritic release of dopamine in the substantia nigra. Nature 289: 537-542.

Chéramy, A., R. Romo, and J. Glowinski (1985) Dopamine release and neuronal activity in the two nigrostriatal systems. In In Vivo Perfusion and Release of Neuroactive Substances, A. Bayón and R. Drucker-Colín, eds., pp. 161-175, Academic, New York.

Chiodo, L. A. (1988) Dopamine-containing neurons in the mammalian central nervous system: Elcctrophysiology and pharmacology. Neurosci. Biobehav. Rev. 12: 49-91.

Chiodo, L. A., and B. S. Bunney (1983) Typical and atypical neuroleptics: Differential effects of chronic administration on the activity of $A 9$ and A10 midbrain dopaminergic neurons. J. Neurosci. 3: 16071619 .

Chiodo, L. A., and G. Kapatos (1987) Mesencephalic dopamine-containing neurons in culture: Morphological and electrophysiological characterization. In The Neurophysiology of Dopamine Systems, L. A. Chiodo and A. S. Freeman, eds., pp. 67-91, Lake Shore Publications, Detroit.

Connor, J. A., and C. F. Stevens (1971) Voltage clamp studies of a transient outward membrane current in gastropod neural somata. $\mathrm{J}$ Physiol. (Lond.) 213: 21-30.

Coombs, J. S., D. R. Curtis, and J. C. Eccles (1957) The interpretation of spike potentials of motoneurones. J. Physiol. (Lond.) 139: 198231.

Crepel, R., and J. Penit-Soria (1986) Inward rectification and low threshold calcium conductance in rat cerebellar Purkinje cells. J. Physiol. (Lond.) 372: 1-23.

Deniau, J. M., C. Hammond, A. Riszk, and J. Feger (1978) Electrophysiological properties of identified output neurons of the substantia nigra pars compacta and pars reticulata: Evidence for the existence of branched projections. Exp. Brain Res. 32: 409-422.

Fallon, J. H., J. N. Riley, and R. Y. Moore (1978) Substantia nigra dopamine neurons: Separate populations project to neostriatum and allocortex. Neurosci. Lett. 7: 157-162.

Fuxe, K., M. Goldstein, T. Hökfelt, and T. H. Joh (1971) Cellular localization of dopamine- $\beta$-hydroxylase and phenylethylamine- $N$ methyl transferase as revealed by immunohistochemistry. Prog. Brain Res. 34: 127-138.

Geffen, L. B., T. M. Jessel, A. C. Cuello, and L. L. Iversen (1976) Release of dopamine from dendrites in rat substantia nigra. Nature 260: 258-260.

Gerfen, C. K., M. Herkenham, and J. Thilbault (1987a) The neostriatal mosaic: II. Patch- and matrix-directed mesostriatal dopaminergic and non-dopaminergic systems. J. Neurosci. 7: 3915-3934.
Gerfen, C. R., K. G. Baimbridge, and J. Thilbault (1987b) The neostriatal mosaic: III. Biochemical and developmental dissociation of patch-matrix mesostriatal systems. J. Neurosci. 7: 3935-3944.

Grace, A. A. (1987) The regulation of dopamine neuron activity as determined by in vivo and in vitro intracellular recordings. In The Neurophysiology of Dopamine Systems. L. A. Chiodo and A. S. Freeman, eds., pp. 1-67, Lake Shore Publications, Detroit.

Grace, A. A. (1988) In vivo and in vitro intracellular recordings from dopamine neurons. Ann. NY Acad. Sci. 537: 51-76.

Grace, A. A., and B. S. Bunney (1980) Nigral dopamine neurons: Intracellular recording and identification using L-DOPA injection combined with fluorescence histochemistry. Science 210:654-656.

Grace, A. A., and B. S. Bunney (1983a) Intracellular and extracellular electrophysiology of nigral dopaminergic neurons. I. Identification and characterization. Neuroscience 10:301-315.

Grace, A. A., and B. S. Bunney (1983b) Intracellular and extracellular electrophysiology of nigral dopaminergic neurons. II. Action potential generating mechansims and morphological correlates. Neuroscience 10 : 317-331.

Grace, A. A., and B. S. Bunney (1984a) The control of firing pattern in nigral dopamine neurons: Single spike firing. J. Neurosci. 4: 28662876.

Grace, A. A., and B. S. Bunney (1984b) The control of firing pattern in nigral dopamine neurons: Burst firing. J. Neurosci. 4: 2877-2890.

Grace, A. A., and B. S. Bunney (1985) Opposing effects of striatonigral feedback pathways on midbrain dopamine cell activity. Brain Res. 333: 271-284.

Grace, A. A., and B. S. Bunney (1986) Induction of depolarization block in midbrain dopamine neurons by repeated administration of haloperidol: Analysis using in vivo intracellular recording. J. Pharmacol. Exp. Ther. 238: 1092-1100.

Grace, A. A., and R. Llinás (1984) Electrophysiology and morphology of four classes of prefrontal neocortical neurons studied in vitro. Soc. Neurosci. Abstr. 10: 739.

Grace, A. A., and R. Llinás (1985) Dehydration-induced morphological artifacts in intracellularly stained neurons: Circumvention using rapid DMSO clearing. Neuroscience 16: 461-475.

Grace, A. A., and S.-P. Onn (1988a) Regulation of sodium and calcium spikes in immunocytochemically identified rat dopamine neurons. Soc. Neurosci. Abstr. 14: 75 .

Grace, A. A., and S.-P. Onn (1988b) Biphasic regulation of activity in immunochemically identified rat midbrain dopamine neurons recorded in vitro. J. Neurosci. Methods 24: 190.

Greenfield, S. A., J. J. B. Jack, A. T. J. Last, and M. French (1988) An electrophysiological action of acetylcholinesterase independent of its catalytic site. Exp. Brain Res. 70: 441-444.

Groves, P. M., and J. C. Linder (1983) Dendro-dendritic synapses in substantia nigra: Descriptions based on analysis of serial sections. Exp. Brain Res. 49: 209-217.

Groves, P. M., C. J. Wilson, S. J. Young, and G. V. Rebec (1975) Selfinhibition by dopaminergic neurons. Science 190: 522-529.

Gulley, R. L., and R. L. Wood (1971) The fine structure of the neurons in the rat substantia nigra. Tissue Cell 3:675-690.

Gustafsson, B., M. Galvan, P. Grafe, and W. Wigstrom (1982) A transient outward current in a mammaliam central neurone blocked by 4-aminopyridine. Nature 299: 252-254.

Guyenet, P. G., and G. K. Aghajanian (1978) Antidromic identification of dopaminergic and other output neurons of the rat substantia nigra. Brain Res. 150: 69-84.

Guyenet, P. G., and J. K. Crane (1981) Non-dopaminergic nigrostriatal pathway. Brain Res. 213: 291-305.

Haas, H. L., and P. B. Reiner (1988) Membrane properties of histaminergic tuberomammillary neurones of the rat hypothalamus in vitro. J. Physiol. (Lond.) 339: 633-646.

Hökfelt, T., O. Johansson, K. Fuxe, M. Goldstein, and D. Park (1976) Immunohistochemical studies on the localization and distribution of monoamine neuron systems in the rat brain. I. Tyrosine hydroxylase in the mes- and diencephalon. Med. Biol. 54: 427-453.

Hornykiewicz, O. (1963) Die topische lokalisation und das verhalten von noradrenalin und dopamin (3-hydroxytyramin) in der substantia nigra des normalin und Parkinsonkranken. Menschen. Wien. Klin. Wochenschr. 75: 309-355.

Hornykiewicz, O. (1966) Dopamine (3-hydroxytyramine) and brain function. Pharmacol. Rev. 18: 925-964.

Jahnsen, H., and R. Llinás (1984a) Electrophysiological properties of guinea-pig thalamic neurones: An in vitro study. J. Physiol. (Lond.) 349: 205-226. 
Jahnsen, H., and R. Llinas (1984b) Ionic basis for the electroresponsiveness and oscillatory properties of guinea pig thalamic neurones in vitro. J. Physiol. (Lond.) 349: 227-247.

Jiminez-Castellanos, J., and A. M. Graybiel (1987) Subdivisions of the dopamine-containing A8-A9-A10 complex identified by their differential mesostriatal innervation of striosomes and extrastriosomal matrix. Neuroscience 23: 223-242.

Jones, E. G. (1975) Varieties and distribution of non-pyramidal cells in the somatic sensory cortex of the squirrel monkey. J. Comp. Neurol. 160: 205-268.

Juraska, J. M., C. J. Wilson, and P. M. Groves (1977) The substantia nigra of the rat: A Golgi study. J. Comp. Neurol. 172: 585-600.

Katz, B. (1949) Les constantes electriques de la membrane du muscle. Arch. Sci. Physiol. 3: 285-300.

Kita, T., H. Kita, and S. T. Kitai (1986) Electrical membrane properties of rat substantia nigra compacta neurons in an in vitro slice preparation. Brain Res. 372: 21-30.

Kitahama, K., L. Denoroy, M. Goldstein, M. Jouvet, and J. Pearson (1988) Immunohistochemistry of tyrosine hydroxylase and phenylethanolamine $\mathrm{N}$-methyltransferase in the human brain stem: Description of adrenergic perikarya and characterization of longitudinal catecholaminergic pathways. Neuroscience 25: 97-111.

Korf, J., M. Zieleman, and B. H. C. Westerink (1976) Dopamine release in the substantia nigra. Nature 260:257-258.

Lacey, M. G., N. B. Mercuri, and R. A. North (1987) Dopamine acts on D2 receptors to increase potassium conductance in neurons of the rat substantia nigra zona compacta. J. Physiol. (Lond.) 392: 397-416.

Lacey, M. G., N. B. Mercuri, and R. A. North (1988) On the potassium conductance increase activated by $\mathrm{GABA}_{\mathrm{B}}$ and dopamine $\mathrm{D}_{2}$ receptors in rat substantia nigra neurones. J. Physiol. (Lond.) 401: 437453.

Lindvall, O., and A. Björklund (1974) The glyoxylic acid fluorescence histochemical method: A detailed account of the methodology for the visualization of central catecholamine neurons. Histochemistry 39 : 97-127.

Lindvall, O., and A. Björklund (1978) Anatomy of the dopaminergic neuron systems in the rat brain. Adv. Biochem. Psychopharmacol. 19: 139-231.

Llinás, R., and M. Sugimori (1980a) Electrophysiological properties of in vitro Purkinje cell somata in mammalian cerebellar slices. J. Physiol. (Lond.) 305: 171-195.

Llinás, R., and M. Sugimori (1980b) Electrophysiological properties of in vitro Purkinje cell dendrites in mammalian cerebellar slices. J. Physiol. (Lond.) 305: 197-213.

Llinás, R., and Y. Yarom (1981a) Electrophysiology of mammalian inferior olivary neurones in vitro. Different types of voltage-dependent ionic conductances. J. Physiol. (Lond.) 315: 549-567.

Llinás, R., and Y. Yarom (1981b) Properties and distribution of ionic conductances generating electroresponsiveness of mammalian inferior olivary neurones in vitro. J. Physiol. (Lond.) 315: 569-584.

Llinás, R., and Y. Yarom (1986) Oscillatory properties of guinea-pig inferior olivary neurones and their pharmacological modulation: An in vitro study. J. Physiol. (Lond.) 376: 163-182.

Llinás, R., S. A. Greenficld, and H. Jahnsen (1984) Electrophysiology of pars compacta cells in the in vitro substantia nigra: A possible mechanism for dendritic release. Brain Res. 294: 127-132.

Matthysse, S. (1973) Antipsychotic drug actions: A clue to the neuropathology of schizophrenia? Fed. Proc. 32: 200-205.

Nakanishi, H., H. Kita, and S. T. Kitai (1987) Intracellular study of rat substantia nigra pars reticulata neurons in an in vitro slice preparation: Electrical membrane properties and response characteristics to subthalamic stimulation. Brain Res. 437: 45-55.

Nedergaard, S., J. P. Bolam, and S. A. Greenfield (1988a) Facilitation of a dendritic calcium conductance by 5-hydroxytryptamine in the substantia nigra. Nature 333: 174-177.

Nedergaard, S., C. Hopkins, and S. A. Greenfield (1988b) Do nigrostriatal neurones possess a discrete dendritic modulatory mechanism? Elcctrophysiological evidence from the actions of amphetamine in brain slices. Exp. Brain Res. 69: 444-448.

Neher, E. (1971) Two fast transient current components during voltage clamp on snail neurons. J. Gen. Physiol. 58: 36-53.

Nowycky, M. C., A. P. Fox, and R. W. Tsien (1985) Three types of neuronal calcium channel with different calcium agonist sensitivity. Nature 316: 440-443.

Ort, C. A., K. J. Futamachi, and J. H. Peacock (1988) Morphology and electrophysiology of ventral mesencephalon nerve cell cultures. Dev. Brain Res. 39: 205-215.

Paxinos, G., and C. Watson (1982) The Rat Brain in Stereotaxic Coordinates, Academic, New York.

Pinnock, R. D. (1984) Hyperpolarizing action of bachlofen on neurons in the rat substantia nigra slice. Brain Res. 332: 337-340.

Pinnock, R. D. (1985) Neurotensin depolarizes substantia nigra dopamine neurons. Brain Res. 338: 151-154.

Preston, R. J., R. A. McCrea, H. T. Chang, and S. T. Kitai (1981) Anatomy and physiology of substantia nigra and retrorubral neurons studied by extra- and intracellular recording and by horseradish peroxidase labeling. Neuroscience $6:$ 331-344.

Renshaw, B. (1942) Effects of presynaptic volleys on spread of impulses over the soma of the motoneuron. J. Neurophysiol. 5: 235243.

Rinvik, E., and I. Grofova (1970) Observations on the fine structure of the substantia nigra in the cat. Exp. Brain Res. 11: 229-248.

Romo, A., A. Chéramy, G. Godeheu, and J. Glowinski (1986) In vivo presynaptic control of dopamine release in the cat caudate nucleusI. Opposite changes in neuronal activity and release evoked from thalamic motor nuclei. Neuroscience 19: 1067-1079.

Ruffieux, A., and W. Schultz (1980) Dopaminergic activation of reticulata neurones in the substantia nigra. Nature 285: 240-241.

Schwartzkroin, P. A., and M. Slawski (1977) Probable calcium spikes in hippocampal neurons. Brain Res. 135: 157-161.

Schwindt, P. C., W. J. Spain, and W. E. Crill (1988) Influence of anomalous rectifier activation on afterhyperpolarizations of neurons from cat sensorimotor cortex in vitro. J. Neurophysiol. 59: 468-481.

Schwyn, R. C., and C. A. Fox (1974) The primate substantia nigra: A Golgi and electron microscopic study. J. Hirnforsch. 16: 95-126.

Shepard, P. D., and B. S. Bunney (1988) Effects of apamin on the discharge properties of putative dopamine-containing neurons in vitro. Brain Res. 463: 380-384.

Silva, N. L., and B. S. Bunney (1988) Intracellular studies of dopamine neurons in vitro: Pacemakers modulated by dopamine. Eur. J. Pharmacol. 149: 307-315.

Snyder, S. H. (1973) Amphetamine psychosis: A model schizophrenia mediated by catecholamines. Am. J. Psychiatry 130: 61-67.

Sternberger, L. A., P. H. Hardy, J. J. Cuculis, and H. G. Meyer (1970) The unlabeled antibody enzyme method of immunocytochemistry. Preparation and properties of soluble antigen-antibody complex (horseradish peroxidase-antihorseradish peroxidase) and its use in identification of spirochetes. J. Histochem. Cytochem. 18: 315-333.

Stewart, W. W. (1978) Functional connections between cells as revealed by dye-coupling with a highly fluorescent naphthalimide tracer. Cell 14: 741-759.

Swanson, L. W., and B. K. Hartman (1975) The central adrenergic system. An immunofluorescence study of the location of cell bodies and their efferent connections in the rat using dopamine- $\beta$-hydroxylase as a marker. J. Comp. Neurol. 163: 467-506.

Tepper, J. M., S. F. Sawyer, and P. M. Groves (1987) Electrophysiologically identified nigral dopaminergic neurons intracellularly labeled with HRP: Light-microscopic analysis. J. Neurosci. 7: 27942806.

Thierry, A. M., J. M. Deniau, and J. Feger (1979) Effects of stimulation of the frontal cortex on identified output VMT cells in the rat. Neurosci. Lett. 15: 103-107.

Thompson, S. H. (1977) Three pharmacologically distinct potassium channels in molluscan neurones. J. Physiol. (Lond.) 265: 465-488.

Waszczak, B. L., and J. R. Walters (1983) Dopamine modulation of the effects of gamma aminobutyric acid on substantia nigra pars reticulata neurons. Science 220: 218-221.

White, F. J., and R. Y. Wang (1983) Comparison of the effects of chronic haloperidol treatment on $\mathrm{A} 9$ and $\mathrm{A} 10$ dopamine neurons in the rat. Life Sci. 32: 983-993.

Wilcox, K., M. J. Gutnick, and G. R. Christoph (1988) Electrophysiological properties of neurons in the lateral habenula nucleus: An in vitro study. J. Neurophysiol. 59: 212-225.

Wong, R. K. S., D. A. Prince, and A. I. Busbaum (1979) Intradendritic recordings from hippocampal neurons. Proc. Natl. Acad. Sci. USA 76: $986-990$.

Yarom, Y., and R. Llinás (1987) Long-term modifiability of anomalous and delayed rectification in guinea pig inferior olivary neurons. J. Neurosci. 7: 1166-1177. 